\title{
Espaços de Banach hereditariamente finitamente decomponíveis
}

\author{
Julio Pereira Neto
}

\author{
DISSERTAÇÃO APRESENTADA \\ AO \\ INSTITUTO DE MATEMÁTICA E ESTATÍSTICA \\ DA \\ UNIVERSIDADE DE SÃO PAULO \\ PARA \\ OBTENÇÃO DE GRAU \\ $\mathrm{DE}$ \\ MESTRE EM MATEMÁTICA \\ Área de Concentração : Análise \\ Orientador : Prof. Dr. Elói Medina Galego
}

SÃO PAULO - MARÇO - 2006 


\section{Espaços de Banach \\ hereditariamente finitamente \\ decomponíveis}

Este exemplar corresponde a redação

final da dissertação devidamente corrigida e defendida por

Julio Pereira Neto

e aprovada pela comissão julgadora.

São Paulo, março de 2006

Banca examinadora :

- Prof. Dr. Elói Medina Galego (Orientador) - IME - USP

- Prof. Dr. Chaim Samuel Hönig - IME - USP

- Prof. Dr. Raymundo Luiz Alencar - ITA 


\section{AGRADECIMENTOS}

- Ao Prof. Elói Medina Galego, não só pela sua paciente orientação, como também pelo incentivo e força nos momentos em que mais precisei.

- A minha esposa Erika e meus filhos Julio e Luis pela compreensão, paciência e amor durante todos os momentos em que estava realizando o trabalho.

- Aos meus pais e irmãs.

- Ao Prof. Valentin Ferenczi pelas dúvidas esclarecidas.

- Ao Carlos pelo apoio com o editor de texto.

- A todos aqueles que direta ou indiretamente contribuíram para a realização deste trabalho.

- Ao Senhor, por ter me dado paz e saúde para trilhar essa caminhada. 


\section{Resumo}

O objetivo desse trabalho é apresentar algumas propriedades e resultados sobre ESPAÇOS DE BANACH HEREDITARIAMENTE FINITAMENTE DECOMPONÍVEIS, introduzido por V. Ferenczi em 1997. Estudaremos suas ligações com espaços de Banach hereditariamente indecomponíveis. Em particular, mostraremos que em um espaço de Banach sobre os reais hereditariamente indecomponível, o quociente do espaço de operadores por espaço de operadores estritamente singulares é um anel de divisão isomorfo ou aos reais, ou aos complexos, ou ao anel de divisão dos quatérnios.

Todos esses resultados foram provados também por V. Ferenczi e publicados em Studia Math. 123 (2) (1997) 135-149.

\section{Abstract}

The aim of this work is to present some properties and results about HEREDITARILY FINITELY DECOMPOSABLE BANACH SPACES, introduced by V. Ferenczi in 1997. We will study their links with hereditarily indecomposable Banach spaces. In particular, we will show that in a real hereditarily indecomposable Banach space, the quotient of the space of operators by the space of strictly singular operators is a division ring isomorphic either to real, complex, or the quaternionic division ring.

All these results were proved by V.Ferenczi and published in Studia Math. 123 (2) (1997) 135-149. 


\section{SUMÁRIO}

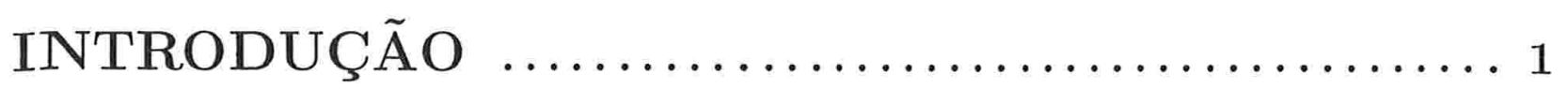

1.PRELIMINARES $\ldots \ldots \ldots \ldots \ldots \ldots \ldots \ldots \ldots \ldots, 4$

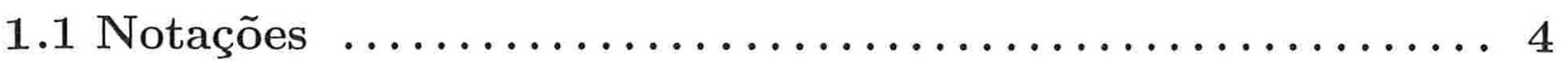

1.2 Definições e resultados $\ldots \ldots \ldots \ldots \ldots \ldots \ldots \ldots \ldots \ldots \ldots$. 5

1.3 Bases em espaços de dimensão infinita $\ldots \ldots \ldots \ldots \ldots \ldots . . \ldots$

1.4 Espaço quociente $\ldots \ldots \ldots \ldots \ldots \ldots \ldots \ldots \ldots \ldots \ldots \ldots \ldots \ldots$

1.5 Operadores estritamente singulares, operadores compactos e operadores de Fredholm .......................... 14

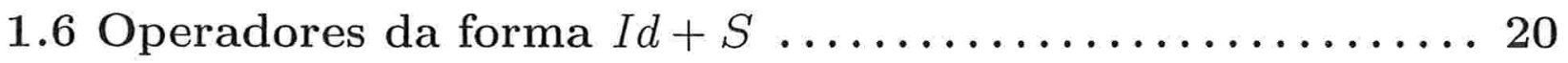

1.7 Espaços de Banach hereditariamente indecomponíveis ... 21

1.8 Álgebras normadas $\ldots \ldots \ldots \ldots \ldots \ldots \ldots \ldots \ldots \ldots \ldots .23$

1.9 Isomorfismo entre os espaços quocientes $E$ e $E_{Y} \ldots \ldots \ldots 26$

2.QUASI-MAXIMALIDADE DE SUBESPAÇOS .. 30

2.1 Quasi-maximalidade $\ldots \ldots \ldots \ldots \ldots \ldots \ldots \ldots \ldots \ldots \ldots \ldots \ldots$

2.2 Soma não direta e subespaços $I d+K$-isomorfos $\ldots \ldots \ldots 30$

2.3 Subespaços quasi-maximais e subespaços $I d+K$-isomorfos 34 
2.4 Quasi-maximalidade de subespaços e operadores estritamente

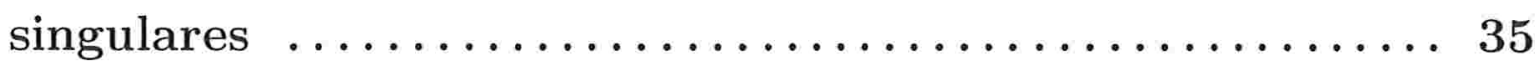

3.ESPAÇOS DE BANACH $H D_{n} \ldots \ldots \ldots \ldots \ldots \ldots \ldots, 37$

3.1 Espaço de Banach hereditariamente finitamente

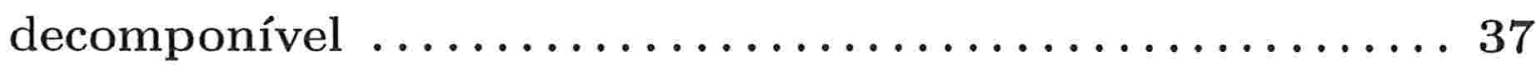

3.2 Somas entre espaços $H D_{n} \ldots \ldots \ldots \ldots \ldots \ldots \ldots \ldots \ldots \ldots$

3.3 Inexistência de sequência básica incondicional em espaços de

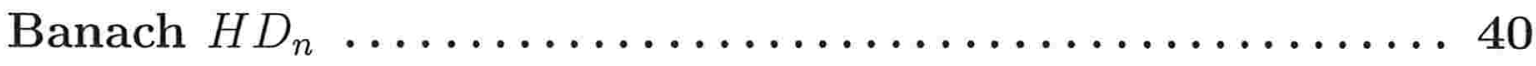

4.ESPAÇOS DE BANACH $H D_{n}$ FUNDAMENTAIS 42

4.1 Espaços $H D_{n}$ fundamentais $\ldots \ldots \ldots \ldots \ldots \ldots \ldots \ldots \ldots \ldots \ldots \ldots$

4.2 Espaços $H D_{n}$ fundamentais, espaços $H D_{n}$ e subespaços quasi-maximais ........................ 43

4.3 Operadores em espaços de Banach $H D_{n}$ fundamentais . . . 44

5.OPERADORES EM ESPAÇOS DE BANACH

COMPLEXO $H D_{n} \ldots \ldots \ldots \ldots \ldots \ldots \ldots \ldots \ldots \ldots$

5.1 Operadores em subespaços de um espaço complexo $H D_{n}$ fundamental ........................... 49

5.2 Dimensão do espaço quociente entre operadores de subespaços de um espaço de Banach complexo $H D_{n}$ e seus operadores estritamente singulares ............. 50 
6.TEORIA ESPECTRAL EM ESPAÇOS $H D_{n} \ldots \ldots 53$

6.1 Definições na teoria espectral em espaços $H D_{n} \ldots \ldots \ldots \ldots 53$

6.2 Caraterísticas do espectro essencial de um operador sobre espaços de Banach ............................. 54

6.3 Operadores semi-Fredholm e Fredholm em espaço de Banach

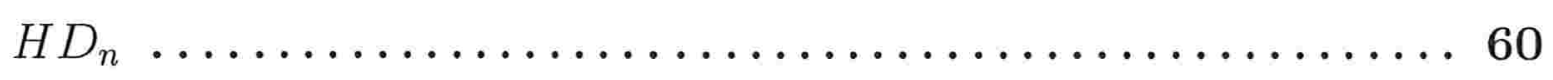

7.O TEOREMA PRINCIPAL $\ldots \ldots \ldots \ldots \ldots \ldots \ldots, 62$

7.1 Operadores em espaço de Banach real H.I. ........... 62

REFERÊNCIAS BIBLIOGRÁFICAS $\ldots \ldots \ldots \ldots 64$ 


\section{INTRODUÇÃO}

Abaixo apresentamos a motivação para se estudar em detalhes o artigo "Hereditarily finitely decomposable Banach spaces". Studia Math. 123(2) 1997 do Prof. Valentin Ferenczi que será o tema principal da dissertação em questão.

DEFINIÇÃO 1. Seja $X$ um espaço de Banach. Uma sequência $\left(e_{n}\right)_{n \in \mathbb{N}}$ de elementos em $X$ é uma base de Schauder para $X$ se para todo $x \in X$, existe uma única sequência de escalares $\left(\lambda_{n}\right)_{n \in \mathbb{N}}$ tal que $x=\sum_{i=1}^{\infty} \lambda_{i} \cdot e_{i}$.

Um problema que ficou em aberto durante anos é o seguinte :

PROBLEMA 2. Todo espaço de Banach separável tem uma base de Shauder?

Este problema foi resolvido negativamente por Enflo em 1973 ([2]). Mazur, no entanto, já havia provado o seguinte :

TEOREMA 3. Todo espaço de Banach de dimensão infinita tem um subespaço de dimensão infinita possuindo uma base de Shauder.

Agora, lembrando a definição abaixo,

DEFINIÇÃO 4. Uma base de Schauder $\left(e_{n}\right)_{n \in \mathbb{N}}$ de um espaço de Banach é incondicional se existe $C \in \mathbb{R}$ tal que $\left\|\sum_{i \in E} \lambda_{i} \cdot e_{i}\right\| \leq C\left\|\sum_{i=1}^{\infty} \lambda_{i} \cdot e_{i}\right\|, \forall E$ finito, $E \subset \mathbb{N}$ e $\forall \lambda_{i} \in \mathbb{K}$

Segue naturalmente, tendo em mente o Problema 2 e o Teorema 3, o seguinte problema :

PROBLEMA 5. Todo espaço de Banach de dimensão infinita possui um subespaço de dimensão infinita com base incondicional ?

Este problema só foi resolvido negativamente em 1993 ([9]) por W.T.Gowers e B.Maurey. Ao resolverem o Problema 5, esses matemáticos também contribuíram para a resolução do seguinte problema que estava em aberto desde 1968: 
PROBLEMA 6. Existe espaço de Banach que seja indecomponível ?

Lembremos que um espaço de Banach $X$ é indecomponível se ele não pode ser escrito como soma direta de dois de seus subespaços de dimensão infinita.

Eles deram, na verdade, a seguinte definição :

DEFINIÇÃO 7. Um espaço de Banach $X$ é hereditariamente indecomponível (H.I.) se nenhum subespaço de $X$ é soma direta de dois subespaços de dimensão infinita.

E provaram,

TEOREMA 8. Existe um espaço H.I.

Ainda mais, o próprio W.T.Gowers ([8]) provou o seguinte teorema de dicotomia em espaços de Banach:

TEOREMA 9. Seja $X$ um espaço de Banach. Então $X$ contém um subespaço com base incondicional ou $X$ contém um subespaço H.I.

A partir desse resultado os espaços H.I. começaram a desempenhar um papel importante na geometria de espaços de Banach.

Um dos objetivos desta dissertação é mostrar que o seguinte resultado de W.T.Gowers e B.Maurey ([9]) sobre espaços H.I. não caracteriza esses espaços.

TEOREMA 10. Seja $X$ um espaço H.I. Então $X$ não tem nenhum subespaço com base incondicional.

Para mostrarmos que existem espaços de Banach que não são H.I. e satisfazem a tese do Teorema 10, estudaremos os espaços $H D_{n}$ introduzidos em 1997 por $V$. Ferenczi no Artigo ([4]).

DEFINIÇÃO 11. Seja $X$ um espaço de Banach e $n \in \mathbb{N}^{*}$. Dizemos que $X$ é $H D_{n}$ se contém um número máximo de $n$ subespaços de dimensão infinita que formam uma soma direta e finita em $X$. 
Segue das Definições 7 e 11, que um espaço de Banach é H.I., se, e somente se, é $H D_{1}$. Mostraremos que a soma direta finita de espaços H.I. é um espaço $H D_{n}$, quando se tem $n$-somandos (Corolário 3.2.3).

Pois bem, os principais resultados ([4]) que apresentaremos em detalhes nesta dissertação são:

TEOREMA 12. (veja respectivamente Teorema 3.3.1 e Teorema 5.1.2) Seja $X$ um espaço $H D_{n}$. Então,

(i) $X$ não contém nenhum subespaço de dimensão infinita com base incondicional;

(ii) para $X=\bigoplus_{i=1}^{n} X_{i}$ um espaço $H D_{n}$ fundamental sobre os complexos temos que todo operador de $Y$, subespaço de dimensão infinita de $X$, tem a forma $\sum_{i \simeq j} \lambda_{i j} p_{i j}+S$ onde $\lambda_{i j}$ é uma constante, $p_{i j}$ é uma projeção e $S$ é estritamente singular.

Indicando por $L(X)$ o conjunto dos operadores lineares em $X$ e por $S(X)$ o conjunto dos operadores estritamente singulares em $X$ foi provado :

TEOREMA 13. (veja Teorema 5.2.1) Se $X$ é um espaço $H D_{n}$ sobre os números complexos, então $\operatorname{dim}(L(X) / S(X)) \leq n^{2}$.

E também, o Teorema Principal,

TEOREMA 14. (veja Teorema 7.1.1) Se $X$ é um espaço de Banach H.I. sobre os números reais então $L(X) / S(X)$ é um anel de divisão isomorfo a $\mathbb{R}$ ou $\mathbb{C}$ ou ao anel dos quatérnios. 


\section{PRELIMINARES}

\subsection{Notações}

Denotaremos por $\mathbb{C}$ o conjunto dos números complexos, $\mathbb{N}$ o conjunto dos números naturais, $\mathbb{R}$ o conjunto dos números reais, $\mathbb{R}_{+}$o conjunto dos números reais não negativos, $\mathbb{R}_{+}^{*}$ o conjunto dos números reais estritamente positivos, $\mathbb{H}$ o anel de divisão dos quatérnios, e $\mathbb{K}$ os corpos $\mathbb{R}$ ou $\mathbb{C}$. Denotaremos por $\overline{\mathbb{A}}$ o fecho do conjunto $\mathbb{A}$ qualquer. vspace- $0.2 \mathrm{~cm}$

O símbolo denota o fim de uma demonstração, e $\sigma$ o vetor nulo de um espaço.

A menos que mencionemos ao contrário, todos os espaços e subespaços de Banach considerados nesta dissertação serão de dimensão infinita e fechado. A dimensão de um espaço de Banach $X$ será denotada por $\operatorname{dim}(X)$.

Sejam $X$ e $Y$ espaços normados. $L(X, Y)$ denotará o espaço de Banach dos operadores lineares contínuos de $X$ em $Y$ com a norma $\|T\|=\sup \{\|T(x)\|:\|x\| \leq 1\}$. Lembremos que um operador linear $T$ é contínuo se, e somente se, existe $M>0$ tal que $\|T(x)\| \leq M\|x\|$, $\forall x \in X$. Denotaremos por $D(T)$ o domínio do operador $T$, por $\operatorname{Im}(T)$ a imagem do operador $T$, isto é, $\operatorname{Im}(T)=\{T(x): x \in X\}$ e por $\operatorname{Ker}(T)$, o Kernel desse operador, ou seja, $\operatorname{Ker}(T)=\{x \in X: T(x)=\sigma\}$. Se $Y=X$, então denotaremos $L(X, Y)=L(X)$.

Sejam $X$ um espaço de Banach e $E$ um subespaço de $X$. Denotaremos por $I d$, o operador identidade $I d: X \rightarrow X$ definido por $I d(x)=x, \forall x \in X$, e por $I_{E, X}$, a inclusão canônica de $E$ em $X$ definida por $I_{E, X}(e)=e, \forall e \in E$. Também denotaremos por $O$, o operador nulo $O: X \rightarrow X$ definido por $O(x)=\sigma, \forall x \in X$.

Um operador linear $T: X \rightarrow Y$ é um isomorfismo se, se somente se, existirem $M$ e $N$ números reais positivos tais que $N\|x\| \leq\|T(x)\| \leq M\|x\|, \forall x \in X$. Nesse caso, dizemos que $X$ e $Y$ são isomorfos e denotaremos $X \simeq Y$.

Dada uma sequência $\left(x_{n}\right)_{n \in \mathbb{N}}$ em um espaço de Banach, usaremos a notação $x_{n} \rightarrow x$ para denotar que a sequência $\left(x_{n}\right)_{n \in \mathbb{N}}$ converge para $x$ quando $n \rightarrow \infty$.

As demais notações serão indicadas no decorrer das definições e resultados desse e dos demais capítulos. 


\subsection{Definições e resultados}

A seguir apresentaremos algumas definições e resultados básicos envolvendo espaços de Banach.

Definição 1.2.1 Seja $X$ um espaço vetorial sobre um corpo $\mathbb{K}$, a aplicação $p: X \rightarrow \mathbb{R}_{+}$é uma semi-norma sobre $X$ se :

- $p(\lambda x)=|\lambda| \cdot p(x), \forall x \in X, \forall \lambda \in \mathbb{K}$

- $p(x+y) \leq p(x)+p(y), \forall x, y \in X$

Se $p(x)=0$ implicar $x=0$, então $p$ é uma norma em $X$.

Definição 1.2.2 Um espaço $X$ é um espaço de Banach se :

- $X$ é um espaço vetorial.

- $X$ é normado.

- X é completo em relação a essa norma.

Definição 1.2.3 Sendo $X$ e $Y$ espaços de Banach, definimos a soma direta externa de $X$ com $Y$ por $X \oplus Y=\{(x, y): x \in X, y \in Y\}$.

Observação 1.2.4 A aplicação $\|\cdot\|_{\infty}: X \oplus Y \rightarrow \mathbb{R}_{+}$definida abaixo é uma norma em. $X \oplus Y$.

$$
\|(x, y)\|_{\infty}=\operatorname{máx}\{\|x\|,\|y\|\}
$$

Proposição 1.2.5 Denotando por $X_{\infty}^{\oplus} Y$ o espaço $X \oplus Y$ da definição 1.2.3 com a norma definida na observação 1.2.4 temos que $X_{\infty}^{\oplus} Y$ é um espaço de Banach.

Prova : verificação imediata 
Proposição 1.2.6 A soma de dois subespaços fechados de um espaço vetorial normado é fechado quando um dos subespaços é de dimensão finita.

Prova: Sugerimos [7], pág.16.

Definição 1.2.7 Sejam $X$ um espaço de Banach e $Y$ um subespaço de $X$. Dizemos que $Y$ é complementado em $X$, se existe um subespaço vetorial $W$ de $X$ tal que :

(i) $Y \cap W=\{\sigma\}$.

(ii) $\forall x \in X, \exists$ ! $y \in Y$ e $\exists$ ! $w \in W: x=y+w$.

(iii) A aplicação linear $p_{1}: X \rightarrow Y$ definida por $p_{1}(x)=y$ é contínua.

(iv) A aplicação linear $p_{2}: X \rightarrow W$ definida por $p_{2}(x)=w$ é contínua.

Observação 1.2.8 Em relação a definição anterior ainda temos que :

(i) $Y$ e $W$ são chamados suplementares topológicos, e escrevemos $X=Y \oplus W$.

(ii) $p_{1}$ é chamada projeção de $X$ sobre $Y$ e vale:
(a) $p_{1}^{2}=p_{1}$
(b) $\left\|p_{1}\right\| \geq 1$
$(\mathrm{c})\left(I d-p_{1}\right)^{2}=I d-p_{1}$

Proposição 1.2.9 Se o subespaço (a princípio não fechado) Y é complementado em um espaço de Banach $X$, então $Y$ é fechado.

Prova : Seja $Y$ o subespaço vetorial complementado do espaço de Banach $X$ da hipótese. Logo, existe $p: X \rightarrow Y$, uma projeção sobre $Y$.

Seja $\left(y_{n}\right)_{n \in \mathbb{N}}$ uma sequência de $Y$ que converge para $x$. Se $p\left(y_{n}\right) \longrightarrow p(x)$ e $p\left(y_{n}\right)=y_{n} \longrightarrow x$, então $x=p(x)$. Logo, $x \in Y$.

Proposição 1.2.10 Sejam $X$ um espaço de Banach e $M$ um subespaço fechado de $X$. Então $M$ é complementado em $X$ se, e somente se, existe um subespaço fechado $N$ de $X$ tal que $X=M \oplus N$. 
Prova : Sugerimos [7], pág.48.

Definição 1.2.11 Seja $R$ um conjunto finito. Então, definimos por $|R|$ a cardinalidade de $R$.

Definição 1.2.12 Dados subespaços $\left(X_{i}\right)_{i \in R}$ de um espaço de Banach $X$, onde $R$ é um conjunto finito de índices, definimos que a soma deles é direta se para todo $i$, a projeção $p_{i}$ de $\sum_{i \in R} X_{i}$ para $X_{i}$ é contínua. Nesse caso, a soma é denotada por $\oplus_{i \in R} X_{i}$ ou $X_{R}$ e para todo operador $T$ sobre $X_{R}$, vamos denotar $T_{\mid R}$ a restrição de $T$ à $X_{R}$ (se $R=\{i\}$, então escrevemos $T_{\mid i}$ para $T_{\left.\right|_{\{i\}}}$ ). Considerando a soma direta $X_{R}$ e dada outra soma direta $X_{R}^{\prime}$, definimos que $X_{R}^{\prime}$ é menor do que $X_{R}$ se existe uma permutação $\delta$ em $R$ tal que para todo $i \in R, X_{i}^{\prime} \subset X_{\delta(i)}$.

Definição 1.2.13 Dois espaços de Banach $X$ e $Y$ são totalmente incomparáveis se nenhum subespaço de $X$ é isomorfo a algum subespaço de $Y$.

Vejamos agora algumas definições e resultados sobre operadores.

Definição 1.2.14 Seja $T: X \rightarrow Y$ um operador linear, onde $X$ e $Y$ são espaços vetoriais normados. O gráfico $G(T)$ de $T$ é o subconjunto $\{(x, T(x): x \in X\}$ de $X \oplus Y$. Se $G(T)$ é fechado em $X \oplus Y$ então $T$ é dito um operador fechado.

Observação 1.2.15 Sejam $X$ e $Y$ espaços vetoriais normados e $T: X \rightarrow Y$ um operador linear. Então, valem as seguintes afirmações:

(i) Té fechado se, e somente se, para qualquer sequência $\left(x_{n}\right)_{n \in \mathbb{N}}$ em $X$, com $x_{n} \rightarrow x$ e $T\left(x_{n}\right) \rightarrow y \in Y$, temos que $y=T(x)$;

(ii) Se T é fechado e injetor então $T^{-1}$ é fechado;

(iii) Se T é fechado então Ker $(T)$ é um subespaço fechado. 
Teorema 1.2.16 (Teorema da aplicação aberta) Seja $T$ uma aplicação linear contínua de um espaço de Banach $X$ sobre um espaço de Banach $Y$. Para todo conjunto aberto $W \subset X, T(W)$ é aberto.

Prova : Sugerimos [10], pág.245.

Corolário 1.2.17 Sejam $X$ e $Y$ espaços de Banach e $T \in L(X, Y)$ bijetora. Então $T$ é bicontínua, isto é, $T^{-1}$ é contínua.

Prova : Sugerimos [10], pág.245.

Teorema 1.2.18 (Teorema do gráfico fechado) Sejam $X$ e $Y$ espaços de Banach $e$ $T: X \rightarrow Y$ uma aplicação linear. Então, $T$ é contínua se, e somente se, o seu gráfico é fechado.

Prova : Sugerimos [10], pág. 251.

Corolário 1.2.19 Sejam $E$ um espaço de Banach, $F$ um espaço normado $e B=$ $\left\{f_{\alpha}: \alpha \in A\right\} \subset L(E, F)$ tal que para todo $x \in E$ tenhamos $\sup \left\{\left\|f_{\alpha}(x)\right\|: \alpha \in A\right\}<+\infty$. Então $\sup \left\{\left\|f_{\alpha}\right\|: \alpha \in A\right\}<+\infty$.

Prova : Sugerimos.[10], pág.217.

Lema 1.2.20 Sejam $X$ e $Y$ espaços de Banach e $T: D(T) \subset X \longrightarrow Y$ um operador fechado. Então $T$ possui um operador inverso contínuo se, e somente se, $T$ é injetor e possui uma imagem fechada.

Prova : Sugerimos [13], pág.17.

Lema 1.2.21 Sejam $X$ e $Y$ espaços de Banach seja $T: X \longrightarrow Y$ um operador fechado com imagem fechada. Se M é um subespaço (não necessariamente fechado) de $X$ tal que 
$M+K e r(T)$ é fechado, então $T(M)$ é fechado. Em particular, se $M$ é fechado e $K e r(T)$ é de dimensão finita, então $T(M)$ é fechado.

Prova: Sugerimos [13], pág. 25.

Lema 1.2.22 Sejam $X$ e $Y$ espaços de Banach e $V$ um subespaço de dimensão finita de $X \oplus Y$. Então $V=V_{X} \oplus V_{Y}$, onde $V_{X}$ e $V_{Y}$ são subespaços de dimensão finita de $X$ e $Y$ respectivamente.

Prova: Imediata.

Lema 1.2.23 Se $V_{1}$ e $V_{2}$ são dois subespaços fechados de um espaço de Banach $X$, de $\operatorname{dim}\left(X / V_{1}\right)=\operatorname{dim}\left(X / V_{2}\right)<\infty$ então existe um isomorfismo de $X$ em $X$ que leva $V_{1}$ sobre $V_{2}$ (o isomorfismo pode ser escolhido sendo o operador identidade sobre $V_{1} \cap V_{2}$ desde que $\left.\operatorname{dim}\left[V_{1} / V_{1} \cap V_{2}\right]<\infty\right)$.

Prova : Sugerimos [13], pág.59.

Proposição 1.2.24 Todo subespaço de dimensão finita de um espaço linear normado é fechado.

Prova : Sugerimos [7], pág. 16.

Proposição 1.2.25 Sejam $X_{1}, X_{2}, \cdots, X_{n}$ subespaços do espaço de Banach $X$. Se $T: X_{1} \underset{\infty}{\oplus} X_{2} \underset{\infty}{\oplus} \cdots \underset{\infty}{\oplus} X_{n} \longrightarrow X_{1}+X_{2}+\cdots+X_{n} \subset X$ é um isomorfismo de $X_{1} \underset{\infty}{\oplus} \cdots \underset{\infty}{\oplus} X_{n}$ sobre $X_{1}^{\infty}+\cdots+X_{n}^{\infty}$, então $X_{1}+\cdots+X_{n}$ é uma soma direta.

Prova: Podemos afirmar que $X_{1} \cap\left[X_{2}+\cdots+X_{n}\right]=\{\sigma\}$ pois, se $x_{1}=x_{2}+\cdots+x_{n}$ e $x_{1} \in X_{1} \cap\left[X_{2}+\cdots+X_{n}\right]$ então $x_{1}-x_{2}-\cdots-x_{n}=\sigma$. E, do isomorfismo temos que $\left(x_{1},-x_{2}, \cdots,-x_{n}\right)=\sigma$.

Seja agora $p_{1}: X_{1}+\cdots+X_{n} \longrightarrow X_{1}$ tal que $p_{1}\left(X_{1}+\cdots+X_{n}\right)=X_{1}$. 


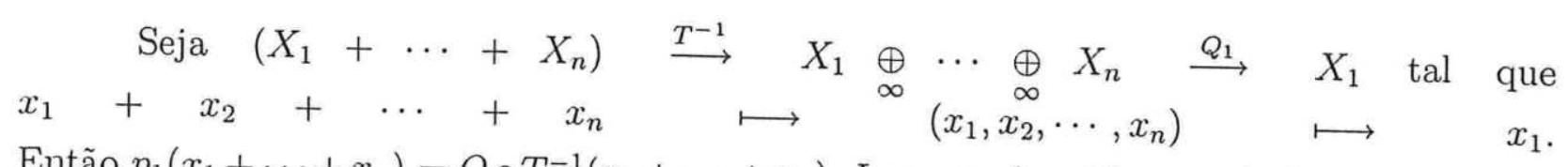
Então $p_{1}\left(x_{1}+\cdots+x_{n}\right)=Q \circ T^{-1}\left(x_{1}+\cdots+x_{n}\right)$. Logo, $p_{1}$ é contínua, pois é uma composta das contínuas $Q$ e $T^{-1}$.

Definição 1.2.26 Uma cisão de um espaço métrico $M$ é uma decomposição $M=A \cup B$, onde $A$ e $B$ são dois subconjuntos abertos disjuntos de $M$. A cisão $M=A \cup B$ diz-se trivial quando um dos abertos é vazio. Um espaço métrico $M$ é dito conexo quando a única cisão possivel de $M$ é a trivial. Um caminho num espaço métrico $M$ é uma aplicação contínua $f:[0,1] \longrightarrow M$. Os pontos $a=f(0)$ e $b=f(1)$ são os extremos do caminho $f$. Um espaço métrico $M$ é dito conexo por caminhos se quaisquer dois pontos de $M$ podem ser unidos por um caminho contido em $M$.

\subsection{Bases em espaços de dimensão infinita}

Nesta seção daremos algumas definições e resultados sobre base em espaços de dimensão infinita. Detalhes sobre essas noções podem ser achados em [12].

Definição 1.3.1 Num espaço de Banach de dimensão infinita $X$, uma sequência $\left(e_{n}\right)_{n \in \mathbb{N}}$ é dita ser uma base de Schauder se todo vetor $x$ em $X$ pode ser escrito unicamente na forma $x=\sum_{i=0}^{\infty} \lambda_{i} e_{i}$, onde $\lambda_{i}^{\prime}$ s são escalares (DEFINIÇÃO 1 da Introdução).

Observação 1.3.2 Nem todo espaço de Banach tem uma base de Schauder de acordo com o contra-exemplo de P. Enflo em 1973 ([2]).

Observação 1.3.3 Foi provado por Mazur que todo espaço de Banach de dimensão infinita tem um subespaço de diminsão infinita possuindo uma base de Schauder (TEOREMA 3 da Introdução). 
Defnição 1.3.4 Num espaço de Banach de dimensão infinita $X$, uma sequência $\left(x_{n}\right)_{n \in \mathbb{N}}$ é dita ser uma base incondicional se existe uma constante $C$ tal que a desigualdade $\left\|\sum_{i \in E} \lambda_{i} x_{i}\right\| \leq C \cdot\left\|\sum_{i=0}^{\infty} \lambda_{i} x_{i}\right\|$, é satisfeita para todo subconjunto finito $E$ de $\mathbb{N}$ e qualquer coeficiente $\lambda_{i}$ (DEFINÇÃO 4 da Introdução).

Observação 1.3.5 Os espaços clássicos $l_{p}$ para $p \geq 1$ contém uma base incondicional, enquanto $L_{1}$ não contém nenhuma.

Definição 1.3.6 Uma sequência $\left(x_{n}\right)_{n \in \mathbb{N}}$ é uma sequência básica (respectivamente uma sequência básica incondicional) se é uma base (respectivamente uma base incondicional) do subespaço fechado gerado por ela, isto é, por $\left[\left(x_{n}\right)_{n \in \mathbb{N}}\right]$.

Proposição 1.3.7 $\left(x_{n}\right)_{n}$ é base de Schauder de um espaço de Banach $X$, se e somente se,

(i) $x_{n} \neq \sigma, \forall n \in \mathbb{N}$;

(ii) $\exists M \in \mathbb{R}_{+}^{*}$ tal que $\left\|\sum_{i=1}^{m} a_{i} x_{i}\right\| \leq M\left\|\sum_{i=1}^{n+p} a_{i} x_{i}\right\|, \forall n, p \in \mathbb{N}$;

(iii) $\left[\left(x_{n}\right)_{n}\right]=X$.

Prova : Sugerimos [12], pág. 2.

Proposição 1.3.8 Seja $X$ um espaço de Banach com base de Schauder $\left(x_{n}\right)_{n \in \mathbb{N}}$. Então os operadores $p_{n}: X \longrightarrow X, n \in \mathbb{N}$ definido por $p_{n}\left(\sum_{i=1}^{\infty} a_{i} x_{i}\right)=\sum_{i=1}^{n} a_{i} x_{i}$ são projeções e $\sup _{n \in \mathbb{N}}\left\|p_{n}\right\|<+\infty$

Prova : Segurimos (EM), pág. 47.

Definição 1.3.9 Seja $\left(x_{n}\right)_{n \in \mathbb{N}}$ uma base de Schauder para o espaço de Banach X. Então $K=\sup _{n \in \mathbb{N}}\left\|p_{n}\right\|$ é chamada constante básica de $\left(x_{n}\right)_{n \in \mathbb{N}}$. 
Proposição 1.3.10 Se $\left(x_{n}\right)_{n \in \mathbb{N}}$ é uma base de Schauder normalizada de um espaço de Banach $X$, então para todo $x=\sum_{i=1}^{\infty} a_{i} x_{i}$ em $X$ temos que $\left|a_{n}\right| \leq 2 K\|x\|, \forall n \in \mathbb{N}$.

Prova : Seja o natural fixado $n \in \mathbb{N}^{*}$. Então temos que $\left|a_{n}\right|=\left|a_{n}\right| \cdot\left\|x_{n}\right\|=\left\|a_{n} x_{n}\right\|=$ $\left\|p_{n}(x)-p_{n-1}(x)\right\| \leq\left\|p_{n}(x)\right\|+\left\|p_{n-1}(x)\right\|=\left\|p_{n}\right\|\|x\|+\left\|p_{n-1}\right\|\|x\| \leq 2 K\|x\|$.

Proposição 1.3.11 Sejam $Y$ um subespaço de dimensão infinita de um espaço de Banach $X, e\left(x_{n}\right)_{n \in \mathbb{N}}$ base de Schauder de $X$. Então para todo inteiro positivo $p$, existe $y \in Y$ com $\|y\|=1$ e da forma $y=\sum_{n=p+1}^{\infty} a_{n} x_{n}$.

Prova: Sugerimos [6], pág. 52.

Lema 1.3.12 Sejam $W$ um subespaço de dimensão infinita de um espaço de Banach $Z$ com base de Schauder $\left(x_{n}\right)_{n \in \mathbb{N}}$. Então para todo inteiro positivo $p$, o subespaço $W_{p+1}$ de $W$ cujos os elementos são da forma $\sum_{i=p+1}^{\infty} a_{i} x_{i}$ para algumas sequências de escalares $\left(a_{i}\right)_{i \in \mathbb{N}}$, é de dimensão inifinita.

Prova : Sejam $W$ um subespaço de dimensão infinita de um espaço de Banach $Z$, e $\left(x_{n}\right)_{n}$ base de Schauder de $X$. Então pela Proposição 1.3.11 segue que para todo inteiro $p$ existe $w_{1} \in W_{p+1} \operatorname{com}\left\|w_{1}\right\|=1$ e da forma $w_{1}=\sum_{i=p+1}^{\infty} a_{i}^{1} x_{i}$.

Seja $n_{1} \in \mathbb{N}$ tal que $\left\|\sum_{i=p+1}^{n_{1}} a_{i}^{1} x_{i}\right\| \geqslant 1 / 2$. Então, novamente pela Proposição 1.3.11, existem $w_{2}=\sum_{i=n_{1}+1}^{\infty} a_{i}^{2} x_{i}$

Seja $n_{2} \in \mathbb{N}$ tal que $\left\|\sum_{i=n_{1}+1}^{n_{2}} a_{i}^{2} x_{i}\right\| \geqslant 1 / 2$. Então continuando por indução encontramos um sequência de números inteiros $p=n_{0}<n_{1}<n_{2}<\cdots$ e vetores $w_{j}=\sum_{i=n_{j-1}+1}^{\infty} a_{i}^{j} x_{i}$.

É claro que $\left(y_{j}\right)_{j \in \mathbb{N}}$ é L.I. com $\left\|\sum_{i=n_{j-1}+1}^{n_{j}} a_{i}^{j} x_{i}\right\| \geq 1 / 2$. 
Proposição 1.3.13 Se $X$ é um espaço de Banach e $\left(x_{n}\right)_{n \in \mathbb{N}}$ base de Schauder de $X$, então $\left(\frac{x_{n}}{\left\|x_{n}\right\|}\right)_{n \in \mathbb{N}}$ é uma base normalizada de $X$ com a mesma constante básica.

Prova: Segue diretamente da Proposição 1.3.7.

Definição 1.3.14 Sejam $X$ um espaço de Banach, $\left(x_{n}\right)_{n \in \mathbb{N}}$ uma sequência básica em $X$, $\left(p_{n}\right)_{n \in \mathbb{N}}$ uma sequência estritamente crescente de inteiros positivos e $\left(a_{n}\right)_{n \in \mathbb{N}}$ uma sequência de escalares. Uma sequência $\left(u_{j}\right)_{j}$ dada por $u_{j}=\sum_{n=p_{j}+1}^{P_{j+1}} a_{n} x_{n}, \operatorname{com} u_{j} \neq 0, \forall j \in \mathbb{N}$, é chamada de base de bloco de $\left(x_{n}\right)_{n \in \mathbb{N}}$.

Proposição 1.3.15 Uma base de bloco $\left(u_{j}\right)_{j \in \mathbb{N}}$ de uma sequência básica $\left(x_{n}\right)_{n \in \mathbb{N}}$ de um espaço de Banach $X$, é uma sequência básica, cuja constante básica é menor ou igual a constante básica de $\left(x_{n}\right)_{n \in \mathbb{N}}$.

Prova: Sugerimos [12], pág. 6.

\subsection{Espaço quociente}

A seguir definimos espaço quociente. Sugerimos também [15], pág. 8.

Definição 1.4.1 Seja $E$ um subespaço do espaço de Banach $X$. Denotamos por $X / E$ o espaço de Banach quociente $\{[x]: x \in X\}$, onde $[x]$ denota a classe de equivalência $x+E$ de $x$ com a norma $\|[x]\|=\inf \{\|x-e\|: e \in E\}$.

Definição 1.4.2 Denotaremos por codim $(E)$ a codimensão do subespaço $E$ no espaço de Banach $X$, isto é, a dimensão do espaço quociente $X / E$. Indicaremos que $E$ tem codimensão finita em $X$ pela notação $\operatorname{codim}(E)<+\infty$.

Notação 1.4.3 Por $Q_{E}$ denotaremos a aplicação quociente canônica de $X$ em $X / E$ definida $\operatorname{por} Q_{E}(x)=[x], \forall x \in X$. 
Proposição 1.4.4 Sejam $X$ e $Y$ espaços de Banach e $T: X \longrightarrow Y$ um operador fechado. Se $\operatorname{Im}(T)$ possui codimensão finita em $Y$ então $\operatorname{Im}(T)$ é um subespaço fechado.

Prova : Sugerimos [13], pág.22.

Proposição 1.4.5 Todo subespaço de codimensão finita de um espaço de Banach X é complementado em $X$.

Prova : Sugerimos [14], pág.373.

Proposição 1.4.6 Sejam $X$ um espaço de Banach e $M$ um subespaço fechado de codimensão finita em $X$. Então.

(i) para qualquer subespaço $V$ de $X$, existe um subespaço de dimensão finita $N$ contido em $V$ tal que $\bar{V}=(\bar{V} \cap M) \oplus N$;

(ii) Se $V$ é denso em $X$, então $V \cap M$ é denso em $M$.

Prova : Sugerimos [13], pág.24.

\subsection{Operadores estritamente singulares, operadores compactos e operadores de Fredholm}

Nesta seção daremos algumas definições e resultados envolvendo operadores estritamente singulares, operadores compactos e operadores de Fredholm.

\subsubsection{Operadores estritamente singulares}

Definição 1.5.1.1 Um operador $S$ de $Z$ para $X$ é dito ser estritamente singular se para todo subespaço $W$ de $Z$, a restrição $\left.S\right|_{W}$ de $S$ para $W$ não é um isomorfismo sobre a imagem. 
Observação 1.5.1.2 Denotamos por $S(X, Y)$ o espaço das aplicações lineares e contínuas $S: X \rightarrow Y$, que são estritamente singulares.

Vamos caracterizar os operadores estritamente singulares.

Proposição 1.5.1.3 Sejam $X$ e $Y$ espaços normados de dimensão infinita. Um operador $T: X \rightarrow Y$ é estritamente singular se, e somente se, $\forall \mathcal{E}>0$, para qualquer $W$ subespaço de dimensão infinita de $X$, existe $x \in W$ tal que $\|T(x)\|<\mathcal{E}\|x\|$.

Prova : Sugerimos [15], pág. 26.

Proposição 1.5.1.4 Sejam $X$ e $Y$ espaços de Banach de dimensão infinita. Seja $S: X \rightarrow Y$ um operador linear e contínuo. $S$ é estritamente singular se, e somente se, para todo subespaço de dimensão infinita $W$ de $X$ e para todo $\mathcal{E}>0$, existe $W^{\prime}$ subespaço de dimensão infinita de $W$, tal que $\left\|\left.S\right|_{W^{\prime}}\right\| \leq \mathcal{E}$.

Prova: Sugerimos [12], pág. 76.

Corolário 1.5.1.5 Sejam $S_{1}$ e $S_{2} \in S(X, Y)$ e $\alpha \in \mathbb{K}$. Então temos $\left(S_{1}+S_{2}\right) \in S(X, Y)$ e $\left(\alpha S_{1}\right) \in S(X, Y)$.

Prova : Sugerimos [15], pág. 27.

Proposição 1.5.1.6 Seja $S$ um operador estritamente singular. Sejam $T$ e U operadores lineares contínuos, para os quais TS e SU estão definidos. Então TS e $S U$ são operadores estritamente singulares.

Prova : Sugerimos [15], pág. 28.

Proposição 1.5.1.7 $S(X, Y)$ é um espaço fechado de $L(X, Y)$.

Prova : Sugerimos [15], pág. 29. 


\subsubsection{Operadores Compactos}

Vejamos agora, a definição e alguns resultados sobre operadores compactos.

Proposição 1.5.2.1 Sejam $X$ e $Y$ espaços normados e $T: X \rightarrow Y$ uma aplicação linear. São equivalentes as seguintes propriedades:

(i) T leva a bola unitária $B$ de $X$ num conjunto relativamente compacto de $Y$, isto é, $\overline{T(B)}$ é compacto.

(ii) T leva os conjuntos limitados de $X$ em conjuntos relativamente compactos de $Y$.

(iii) Toda sequência limitada $\left(x_{n}\right)_{n \in \mathbb{N}}$ de pontos em $X$ contém uma subsequência $x_{n_{k}}$ tal que a sequência $\left(T\left(x_{n_{k}}\right)\right)_{k \in \mathbb{N}}$ é convergente em $Y$.

Prova: Sugerimos [11], pág. 302.

Definição 1.5.2.2 Dizemos que um operador linear $T: X \rightarrow Y$ é compacto se satisfaz as condições equivalentes citadas na proposição anterior.

Proposição 1.5.2.3 Sejam $X$ e $Y$ espaços normados e $T: X \rightarrow Y$ um operador linear $e$ contínuo, cuja imagem tem dimensão finita. Então $T$ é compacto.

Prova: Sugerimos [11] pág. 303.

Definição 1.5.2.4 Sejam $X$ e $Y$ espaços normados, $T$ e $\left(T_{n}\right)_{n \in \mathbb{N}}$ um sequência de operadores lineares. Dizemos que $T_{n}$ converge uniformemente para $T$ se $\forall \mathcal{E}>0, \exists n_{0} \in \mathbb{N}$ tal que $\forall x \in X,\|x\| \leq 1$ temos $\left\|T_{n}(x)-T(x)\right\| \leq \mathcal{E}, \forall n \geq n_{0}$.

Proposição 1.5.2.5 Sejam $X$ um espaço normado, $Y$ um espaço de Banach e $T_{n}: X \rightarrow Y$, $n \in \mathbb{N}$, uma sequência de operadores compactos tal que $T_{n}$ converge uniformemente para $T$. Então T é compacto.

Prova: Sugerimos [11], pág. 312. 
É bem conhecido (veja [12], pág. 76) que existem operadores estritamente singulares que não são operadores compactos, no entanto, temos:

Proposição 1.5.2.6 Sejam $X$ e $Y$ espaços normados de dimensão infinita. Se $K: X \rightarrow Y$ é um operador compacto, então $K$ é estritamente singular.

Prova : Sugerimos [15], pág. 31.

Proposição 1.5.2.7 Sejam X e $Y$ espaços de Banach de dimensão infinita. Assuma que $T: X \rightarrow Y$ seja um operador tal que a restrição de $T$ para nenhum subespaço de $X$ de codimensão finita é um isomorfismo. Então, para todo $\mathcal{E}>0$, existe um subespaço $Z$ de dimensão infinita de $X$ tal que $\left.T\right|_{z}$ é compacto $e\left\|T_{\left.\right|_{Z}}\right\| \leq \mathcal{E}$.

Prova: Sugerimos [12], pág. 76.

Observação 1.5.2.8 Sejam $X$ e $Y$ espaços de Banach de dimensão infinita e $T$ um operador linear contínuo de $X$ e $Y$. Se para algum subespaço $X_{0}$ de $X$, de codimensão finita, a restrição de $T$ à $X_{0}$ é um isomorfismo sobre a imagem, então $T(X)$ é fechado em $Y$.

\subsubsection{Operadores de Fredholm}

Vamos apresentar agora algumas definições e um resultado sobre operadores de Fredholm.

Definição 1.5.3.1 Sejam $X$ e $Y$ espaços de Banach e $T \in L(X, Y)$ um operador com imagem fechada. Definimos o índice do núcleo e o índice de deficiência de $T$, respectivamente por :

$\alpha(T)=\operatorname{dim}(\operatorname{ker}(T)) \quad$ e $\quad \beta(T)=\operatorname{dim}(Y / \operatorname{Im}(T))=\operatorname{dimcoker}(T)$. 
Definição 1.5.3.2 Sejam $X$ e $Y$ espaços de Banach. Então um operador $T \in L(X, Y)$ é dito ser semi-Fredholm superior se sua imagem $\operatorname{Im}(T)$ é fechada e $\alpha(T)<\infty$. O conjunto de todos os operadores semi-Fredholm superior é denotado por $\Phi_{+}(X, Y)$. Um operador $T \in L(X, Y)$ é dito ser semi-Fredholm inferior se $\beta(T)<\infty$ (neste caso, $\operatorname{Im}(T)$ é fechado pela Proposição 1.4.4). O conjunto de todos os operadores semi-Fredholm inferior é denotado por $\Phi_{-}(X, Y)$.

Definição 1.5.3.3 Sejam $X$ e $Y$ espaços de Banach e $T \in L(X, Y)$ um operador semiFredholm superior ou semi-Fredholm inferior. Definiremos o índice de :

$$
T \in \Phi_{+}(X, Y) \cup \Phi_{-}(X, Y) \text { por } i(T)=\alpha(T)-\beta(T)
$$

Definição 1.5.3.4 Sejam $X$ e $Y$ espaços de Banach e $T \in L(X, Y)$. Então $T$ é chamado operador de Fredholm se $i(T)<\infty$, ou equivalentemente, se $T$ é um operador semi-Fredholm superior e semi-Fredholm inferior.

O conjunto de todos os operadores Fredholm é denotado por $\Phi(X, Y)$.

Proposição 1.5.3.5 Sejam $X$ e $Y$ espaços de Banach e $T \in L(X, Y)$ um operador com imagem fechada para o qual $\alpha(T)<+\infty$. Seja $S: X \rightarrow Y$ um operador estritamente singular. Então $\alpha(T+S)<\infty, T+S$ tem imagem fechada e $i(T+S)=i(T)$.

Prova : Sugerimos [12], pág. 79.

Proposição 1.5.3.6 Sejam $T: X \longrightarrow Y$ um operador Fredholm e $S: X \longrightarrow Y$ um operador tal que $\operatorname{dim}(T(X))<+\infty$. Então $T+S$ é um operador Fredholm e $i(T+S)=i(T)$.

Prova : Sugerimos [12], pág.77.

Observação 1.5.3.7 Denotando por $L$ a classe de todos os operadores lineares contínuos entre espaços de Banach arbitrários podemos definir que um ideal de operadores $U$ é uma subclasse de $L$ tal que dados espaços de Banach $X$ e $Y$ as componentes $U(X, Y)=U \cap L(X, Y)$, 
satisfazem as seguintes condições:

(i) $I d_{E} \in U$, onde $E$ denota o espaço de Banach de dimensão 1;

(ii) Se $S_{1}, S_{2} \in U(X, Y)$ então $S_{1}+S_{2} \in U(X, Y)$;

(iii) Sejam $X_{0}$ e $Y_{0}$ espaços de Banach de $T \in L\left(X_{0}, X\right), S \in U(X, Y)$ e $R \in L\left(Y, Y_{0}\right)$ então $R S T \in U\left(X_{0}, Y_{0}\right)$.

Se $X=Y$ denotamos a componente $U(X, Y)$ por $U(X)$.

Definição 1.5.3.8 Sejam $X$ um espaço de Banach e os operadores $T \in L(X)$. Então, definimos a álgebra de Calkin por $L(X) / K(X)$ onde $K(X)$ é o ideal de todos os operadores compactos sobre o espaço de Banach $X$. Denotaremos por $Q$, a aplicação quociente $Q: L(X) \longrightarrow L(X) / K(X)$ definida por $Q(T)=[T], \forall T \in L(X)$.

Proposição 1.5.3.9 Seja $X$ um espaço de Banach e $T: X \longrightarrow Y$. Um operador $T \in L(X)$ é Fredholm se, e somente se $[T]$ é invertível na álgebra de Calkin $L(X) / K(X)$.

Prova : Sugerimos [13], pág.47.

Proposição 1.5.3.10 Sejam $X$ e $Y$ espaços de Banach e $T: X \longrightarrow Y$, um operador para o qual $i(T)$ é definido. Existe um número $\lambda(T)>0$ tal que se $S: X \longrightarrow Y$ satisfaz $\|S\|<\lambda(T)$ então :

(i) $\alpha(T+S) \leq \alpha(T)$;

(ii) $T+S$ tem uma imagem fechada e $\beta(T+S) \leq \beta(T)$;

(iii) $i(T+S)=i(T)$.

Prova: Sugerimos [12], pág. 78. 


\subsection{Operadores da forma $I d+S$}

Veremos nessa seção um resultado que caracteriza os operadores da forma $(I d+S)$ e definições decorrentes.

Proposição 1.6.1 Sejam $Z$ um subespaço de um espaço de Banach $X$ e $A \in L(Z, X)$. Se $A$ é da forma $I d+S$, onde $S$ é estritamente singular, então $A$ é um isomorfismo sobre algum subespaço de codimensão finita de $Z$.

Prova : Seja $A \in L(Z, X)$, onde $Z$ é um subespaço de dimensão infinita do espaço de Banach $X$ tal que a restrição de $A$ para algum subespaço $Z^{\prime}$ de codimensão finita de $Z$ não é um isomorfismo.

Então, pela Proposição 1.5.2.7 temos que para todo $\mathcal{E}>0$ existe um subespaço $Z^{\prime \prime}$ de $Z$ tal que $\left.A\right|_{Z^{\prime \prime}}$ é compacto. E mais pela Proposição 1.5.2.6, $\left.A\right|_{Z^{\prime \prime}}$ é estritamente singular.

Assim, se considerarmos $A$ na forma $(I d+S)$ onde $S$ é estritamente singular teremos : $\left.A\right|_{Z^{\prime \prime}}=\left.I d\right|_{Z^{\prime \prime}}+\left.S\right|_{Z^{\prime \prime}}$, ou melhor $\left.A\right|_{Z^{\prime \prime}}-\left.S\right|_{Z^{\prime \prime}}=\left.I d\right|_{Z^{\prime \prime}}$.

Segue pelo Corolário 1.5.1.5, que $\left.I d\right|_{Z^{\prime \prime}}$ é estritamente singular, o que é uma contradição, pois o operador identidade é bijetor e bicontínuo e portanto, um isomorfismo sobre a sua imagem.

Logo, se $A$ é da forma $(I d+S)$, então $A$ é um isomorfismo sobre algum subespaço de codimensão finita.

Definição 1.6.2 Se cada operador $A \in L(Z, X)$ é um isomorfismo sobre $Z$ na forma da Proposição 1.6.1, dizemos que A é um $(I d+S)$-isomorfismo.

Logo se existe $A \in L(Z, X),(I d+S)$-isomorfismo, então o isomorfismo inverso $A^{-1}$ de $A$ definido sobre $A(Z)$ satisfaz $A^{-1}-I d=(I d-A) A^{-1}$. Sendo assim temos: $A^{-1}=I d+(I d-A) A^{-1}$, portanto $A^{-1}$ é um $(I d+S)$-isomorfismo sobre $A(Z)$. 
Definição 1.6.3 Dois subespaços $Y$ e $Z$ de um espaço de Banach $X$ são ditos serem $(I d+S)$-isomorfos se existe um $(I d+S)$-isomorfismo de $Y$ para $Z$.

Definição 1.6.4 Substituindo o operador estritamente singular $S$ por um operador compacto $K$ na Proposição 1.6.1, também se define $A$ como $(I d+K)$-isomorfismo e consequentemente na definição 1.6.3, subespaços $(I d+K)$-isomorfos.

Lembremos a Proposição 1.5.2.6 que afirma que todo operador compacto é estritamente singular.

\subsection{Espaços de Banach hereditariamente indecomponíveis ( ou H.I.)}

O objetivo dessa seção é definir e apresentar resultados que caracterizam os espaços de Banach hereditariamente indecomponíveis.

Definição 1.7.1 Um espaço de Banach de dimensão infinita que pode ser escrito como uma soma de pelo menos dois subespaços de dimensão infinita é dito ser decomponível.

Definição 1.7.2 Um espaço de Banach de dimensão infinita é indecomponível se não pode ser escrito como soma direta de seus subespaços de dimensão infinita.

Definição 1.7.3 Um espaço de Banach de dimensão infinita é hereditariamente indecomponivel ( ou H.I.) se nenhum subespaço dele é soma direta de dois subespaços de dimensão infinita, ou seja, não admite subespaço de dimensão infinita decomponível (DEFINIÇÃO 7 da Introdução).

Observação 1.7.4 Gowers e Maurey, em 1993, construíram o primeiro espaço de Banach H.I. chamado $X_{G M}$, veja [9] (TEOREMA 8 da Introdução). 
Definição 1.7.5 Sejam $Y$ e $Z$ subespaços fechados de dimensão infinita,

$$
\gamma(Y, Z)=\inf \{\|z-y\|:\|z\|=\|y\|=1, y \in Y \text { e } z \in Z\}
$$

é definido como sendo o ângulo entre os subespaços $Y$ e $Z$.

Lema 1.7.6 Sejam $Y$ e $Z$ subespaços fechados de dimensão infinita de um espaço de Banach $X$, tal que $Y \cap Z=\{\sigma\}$. Então $Y+Z$ é fechado, se e somente se, $\gamma(Y, Z)>0$.

Prova: Sugerimos [15], pág. 12.

Proposição 1.7 .7 (Caracterização geométrica dos espaços de Banach H.I.) Seja X um espaço de Banach. $X$ é H.I. se, e somente se, para quaisquer $Y$ e $Z$ subespaços fechados de dimensão infinita de $X$, e para qualquer $\mathcal{E}>0$, existe $y \in Y$ e $z \in Z$ com $\|y\|=\|z\|=1$ tal que $\|y-z\|<\mathcal{E}$.

Prova : Sugerimos [15], pág.14.

Muitos resultados conhecidos nos espaços H.I. são sobre operadores em espaços complexos H.I.. Em particular, Gowers e Maurey mostraram em [9] que se X é um espaço complexo H.I., então todo operador sobre $X$ é a soma de um operador estritamente singular com uma múltipla identidade.

Teorema 1.7.8 (Generalização do resultado de Gowers e Maurey) Sejam X um espaço complexo H.I. e $Y$ um subespaço de $X$. Então cada operador de $Y$ para $X$ é da forma $\lambda I_{Y, X}+S$, onde $\lambda$ é uma constante complexa, a aplicação $I_{Y, X}$ é a inclusão conônica de $Y$ para $X$, e $S$ é um operador estritamente singular.

Prova : Sugerimos [3]. 


\section{8 Álgebras normadas}

Nesta seção apresentaremos alguns conceitos e resultados que serão úteis na prova do Teorema Principal (Teorema 7.1.1 do Capítulo 7).

Definição 1.8.1 Uma álgebra normada sobre um corpo $\mathbb{K}$ é um espaço vetorial normado $A$, sobre $\mathbb{K}$, no qual definimos uma operação de multiplicação interna, satisfazendo os seguintes axiomas:

(i) $(x y) z=x(y z), \forall x, y, z \in A$;

(ii) $x(y+z)=x y+x z$ e $(y+z) x=y x+z x, \forall x, y, z \in A$;

(iii) $\lambda(x y)=(\lambda x) y=x(\lambda y), \forall x, y, z \in A$ e $\forall \lambda \in \mathbb{K}$;

(iv) $\|x y\| \leq\|x\| \cdot\|y\|, \forall x, y \in A$.

Observação 1.8.2 Considerando a definição anterior temos que:

(i) Se $\mathbb{K}=\mathbb{R}, A$ é chamada álgebra normada real, e se $\mathbb{K}=\mathbb{C}$, álgebra normada complexa;

(ii) Uma álgebra normada é comutativa se $x y=y x \forall x, y \in A$;

(iii) Se A é um espaço vetorial completo em relação à norma $\|\cdot\|$ e é uma álgebra normada, então dizemos que A é uma Álgebra de Banach.

Definição 1.8.3 Um elemento e de uma álgebra normada A é chamado unidade de $A$ se, e somente se, $e \neq 0$, ex $=x e=x, \forall x \in A \quad e\|e\|=1$.

Observação 1.8.4 Em relaçã a Definição 1.8.3 temos que :

(i) A é uma álgebra normada com unidade se existe em A o elemento unidade;

(ii) É posssível mostrar que o elemento unidade quando existe é único;

(iii) Sendo A uma álgebra normada com unidade, denotaremos o elemento unidade por 1. 
Definição 1.8.5 Sejam A uma álgebra normada com unidade e $x \in A$. Dizemos que $x$ é invertivel se existe $y \in A$ tal que $x y=1$ e $y x=1$. O elemento y será chamado inverso de $x$ e o denotaremos por $x^{-1}$.

Definição 1.8.6 Uma álgebra normada com divisão é uma álgebra normada $A$ com unidade tal que todo elemento não nulo é invertivel.

Teorema 1.8.7 (Caso Complexo do Teorema de Gelfand-Mazur) Seja A uma álgebra de Banach complexa com divisão, então $A$ é isométricamente isomorfo a $\mathbb{C}$.

Prova : Sugerimos [15], pág.16.

Definição 1.8.8 Seja A uma álgebra real. A complexificação $A_{C}$ de $A$ é o conjunto $A \times A$ para o qual definimos, para todo $a, b, c, d \in A, \alpha, \beta \in \mathbb{R}$, as seguintes operações:

(i) $(a, b)+(c, d)=(a+c, b+d)$;

(ii) $(\alpha+\beta i)(a, b)=(\alpha a-\beta b, \alpha b+\beta a)$;

(iii) $(a, b)(c, d)=(a c-b d, a d+b c)$.

Definição 1.8.9 É fácil verificar que $A_{C}$ possui estrutura de álgebra complexa e que a aplicação $a \rightarrow(a, 0)$ é um monomorfismo de $A$ em $A_{C}$.

Lembramos que para $A$ e $B$ álgebras sobre um corpo $\mathbb{K}$ um monomorfismo de $A$ em $B$ é um homomorfismo injetivo de $A$ em $B$. Relembrando que homomorfismo de $A$ em $B$ é uma aplicacção $\Phi \in L(A, B)$ tal que $\Phi(x y)=\Phi(x) \cdot \Phi(y), \forall x, y \in A$.

Definição 1.8.10 Seja A uma álgebra normada real, então temos:

(i) A tem unidade se e somente se $A_{C}$ tem unidade. Se 1 é a unidade de $A$, então $(1,0)$ é a unidade de $A_{C}$.

(ii) $a \in A$ é invertivel se e somente se $(a, 0) \in A_{C}$ é invertível, $e(a, 0)^{-1}=\left(a^{-1}, 0\right)$. 
Prova : Sugerimos [1], pág. 69.

Definição 1.8.11 Sejam $1, i, j, k$ a base usual de vetores do $\mathbb{R}^{4}$, isto é, $1=(1,0,0,0), i=$ $(0,1,0,0), j=(0,0,1,0), k=(0,0,0,1)$. A álgebra dos quatérnios sobre $\mathbb{R}$, denotada por $\mathbb{H}$ é o espaço vetorial $\mathbb{R}^{4}$ com o produto interno definido sobre os vetores da base da seguinte forma:

$$
\begin{array}{r}
1^{1}=1, \quad 1 i=i 1=i, \quad 1 j=j 1=j, \quad 1 k=k 1=k, \\
i^{2}=j^{2}=k^{2}=-1, \quad i j=-j i=k, \quad j k=-k j=i, \quad k i=-i k=j .
\end{array}
$$

Observação 1.8.12 Estendendo a multiplicação anterior, por linearidade, para as combinações lineares dos elementos da base e considerando a norma definida por $|x|=\left(\sum_{m=1}^{4} x_{m}^{2}\right)^{\frac{1}{2}}$, onde $x=\left(x_{1}, x_{2}, x_{3}, x_{4}\right) \in \mathbb{H}$, é possível mostrar através de cálculos rotineiros que $\mathbb{H}$ é álgebra sobre $\mathbb{R}$, e que $|x y|=|x||y| \forall x, y \in \mathbb{H}$, e portanto que $\mathbb{H}$ é uma álgebra normada sobre $\mathbb{R}$.

Observação 1.8.13 O elemento 1 é a unidade de $\mathbb{H}$, pois dado $x=\left(x_{1}, x_{2}, x_{3}, x_{4}\right) \in \mathbb{H}$ temos:

$1 x=(1,0,0,0)\left(x_{1}, x_{2}, x_{3}, x_{4}\right)=(1+0 i+0 j+0 k)\left(x_{1}+x_{2} i+x_{3} j+x_{4} k\right)=x_{1}+x_{2} i+x_{3} j+x_{4} k=x$ e de forma análoga podemos mostrar que $x 1=x$.

Observação 1.8.14 Sejam $x=\left(x_{1}, x_{2}, x_{3}, x_{4}\right)$ e $x^{*}=\left(x_{1},-x_{2},-x_{3},-x_{4}\right)$ em $\mathbb{H}$. Então $x x^{*}=\left(x_{1}, x_{2}, x_{3}, x_{4}\right)\left(x_{1},-x_{2},-x_{3},-x_{4}\right)=\left(x_{1}+x_{2} i+x_{3} j+x_{4} k\right)\left(x_{1}-x_{2} i-x_{3} j-x_{4} k\right)=$ $1|x|^{2}+0 i+0 j+0 k=|x|^{2}$. Assim, sendo $x \neq 0$, podemos considerar o elemento $x^{-1}=\frac{1}{|x|^{2}} x^{*}$ tal que $x x^{-1}=x \cdot\left(\frac{1}{|x|^{2}} x^{*}\right)=\frac{1}{|x|^{2}}\left(x x^{*}\right)=1$. De forma análoga podemos mostrar que $x^{-1} x=1$. Logo $\mathbb{H}$ é uma álgebra normada sobre $\mathbb{R}$ com divisão.

Teorema 1.8.15 (caso real do Teorema de Gelfand-Mazur) Seja A uma álgebra normada real, com divisão. Então A é isomorfo a $\mathbb{R}, \mathbb{C}$ ou $\mathbb{H}$.

Prova : Sugerimos [15], pág.23. 


\subsection{Isomorfismo entre os espaços quocientes $E$ e $E_{Y}$}

\subsubsection{O espaço quociente $E_{Y}$}

No que segue na Seção 1.9, $X$ será espaço de Banach de dimensão infinita, e o conjunto dos subespaços fechados de dimensão infinita de $X$ será denotado por $G_{X}$. Também no que segue consideramos para cada $Y \in G_{X}$ a aplicação $\|\cdot\|_{Y}$ definida sobre $L(Y, X)$ por $\|T\|_{Y}=\sup _{Z^{\prime} \subset Y Z \subset \subset Y}\left\|\left.T\right|_{Z^{\prime}}\right\|$.

Observação 1.9.1.1 Em relação a norma acima mencionada temos:

(i) $\|T\|_{Y}=0$ se, e somente se, $T$ é estritamente singular;

(ii) se $X$ é H.I., então para todo $T \in L(Y, X)$ e para todo $Z \subset Y$ temos : $\|T\|_{Y}=\inf _{Z^{\prime} \subset Z}\left\|\left.T\right|_{Z^{\prime}}\right\|$.

Prova : Sugerimos [15], respectivamente pág. 41 e 46 para as observações $i$ ) e ii).

A seguir nesta seção (1.9), $X$ será um espaço de Banach hereditariamente indecomponível.

Definição 1.9.1.2 Para cada $Y \in G_{X}$ denotamos por $E_{Y}$ o espaço quociente de $L(Y, X)$ pelo Kernel da aplicação $\|\cdot\|_{Y}$, isto é, $E_{Y}=L(Y, X) / S(Y, X)$. Denotamos por $\alpha_{Y}$ um elemento de $E_{Y}$, e para todo $T \in L(Y, X)$, denotamos por $\bar{T}$ a classe de $T$ em $E_{Y}$, isto é $\bar{T}=\{U \in L(Y, X): U-T \in S(Y, X)\}$. Consideramos a seguinte aplicação \|\|$\cdot\|\|_{Y}: E_{Y} \longrightarrow \mathbb{R}_{+}$dada por $\left\|\alpha_{Y}\right\|\left\|_{Y}=\right\| T \|_{Y}, \forall T \in \alpha_{Y}$, que é uma norma em $E_{Y}$.

Definição 1.9.1.3 Sejam $Z, Y \in G_{X}$ tal que $Z$ é um subespaço de $Y$. Existe $Y^{\prime}$ subespaço de $Y$ tal que $Y^{\prime}=\left(I_{Z}+S\right)(Z)$ onde $I_{Z}+S$ é um isomorfismo. Definimos então a seguinte aplicação de $E_{Y}$ em $E_{Z}: \quad P_{Y Z}: E_{Y} \longrightarrow E_{Z}$ tal que $\bar{T} \longmapsto \overline{T\left(I_{Z}+S\right)}$. 
Lema 1.9.1.4 Sejam $Y$ e $Z$ em $G_{X}$ tal que $Z$ é um subespaço de $Y$. Então $P_{Y Z}$ é uma isometria linear.

Prova : Sugerimos [15], pág.50.

\subsubsection{O espaço quociente $E$}

Definição 1.9.2.1 Definimos em $X$, o espaço vetorial normado $l_{\infty}\left(\left(E_{Y}\right)_{Y \in G_{X}}\right)=\left\{\left(\alpha_{Y}\right)_{Y \in G_{X}} / \exists M \in \mathbb{R}_{+}^{*}: \mid\left\|\alpha_{Y}\right\|_{Y} \leq M, \forall Y \in G_{X}\right\}$, onde : $\left\|\left(\alpha_{Y}\right)_{Y}\right\|=\sup _{Y \in G_{X}}\left\|\alpha_{Y}\right\| \|_{Y}$.

Proposição 1.9.2.2 Denotamos por $\Omega=\left\{\left(\alpha_{Y}\right)_{Y \in G_{X}} \in l_{\infty}\left(\left(E_{Y}\right)_{Y \in G_{X}}\right)\right.$ se existe $Y_{0} \in G_{X}$ tal que para todo $Y$ subespaço de $Y_{0}$ temos $\left.\alpha_{Y}=P_{Y_{0} Y}\left(\alpha_{Y_{0}}\right)\right\}$. Afirmamos que $\Omega$ é um espaço linear.

Prova: Sugerimos [15], pág.57.

Definição 1.9.2.3 Dado $\left(\alpha_{Y}\right)_{Y \in G_{X}} \in \Omega$ segue do Lema 1.9.1.4 que $\left\|\left|\alpha_{Y}\left\|\left.\right|_{Y}=\right\|\right| \alpha_{Y_{0}} \mid\right\|_{Y_{0}}$, para todo $Y$ subespaço de $Y_{0}$, pois $\alpha_{Y}=P_{Y_{0} Y}\left(\alpha_{Y_{0}}\right)$ e $P_{Y_{0} Y}$ é uma isometria. Assim podemos definir a seguinte aplicação em $\Omega$ :

$\Psi: \Omega \longrightarrow \mathbb{R}_{+}$definida por $\left(\alpha_{Y}\right)_{Y} \longmapsto \Psi\left(\left(\alpha_{Y}\right)_{Y}\right)=\left\|\alpha_{Y_{0}}\right\| \mid$.

Observação 1.9.2.4 Denotamos por $\Gamma=\left\{\left(\alpha_{Y}\right)_{Y \in G_{X}} \in \Omega: \Psi\left(\left(\alpha_{Y}\right)_{Y}\right)=0\right\}$ o subespaço fechado de $\Omega$.

Prova : Sugerimos [15], pág.60.

Definição 1.9.2.5 Definimos o espaço quociente $E=\Omega / \Gamma$ tal que $\alpha=\overline{\left(\alpha_{Y}\right)_{Y \in G_{X}}} \in E$ onde a aplicação $\|\cdot\|: E \longrightarrow \mathbb{R}_{+}$dada por $\|\alpha\|=\Psi\left(\left(\alpha_{Y}\right)_{Y}\right), \forall\left(\alpha_{Y}\right)_{Y} \in \alpha$ é uma norma em $E$ (veja [15], pág 61). 


\subsubsection{Isometria entre $E$ e $E_{Y}$}

Os próximos resultados ajudam a provar no Capítulo 7 que o espaço $E_{Y}$ é isometricamente isomorfo aos espaços $\mathbb{R}, \mathbb{C}$ e $\mathbb{H}$.

Definição 1.9.3.1 Sejam $\alpha$ e $\beta \in E$. Então definimos $\alpha \beta \in E$ por $\overline{\left(\alpha_{Y_{0}} \otimes \beta_{Z \subseteq Z_{1}}\right)}$ onde $Y_{0}, Z_{1} \in G_{X}$ são tais que $\alpha_{Y}=P_{Y_{0} Y}\left(\alpha_{Y_{0}}\right), \forall Y$ subespaço de $Y_{0}$ e $\beta_{z} \in E_{Z Y_{0}}, \forall Z$ subespaço de $Z_{1}$.

Proposição 1.9.3.2 O espaço E com a multiplicação dada na definição 1.9.3.1 e norma definida em 1.9.2.5 possui estrutura de álgebra normada com unidade.

Prova : Sugerimos [15], pág.67.

Definição 1.9.3.3 Seja $X$ um espaço de Banach H.I. . Para cada $Y \in G_{X}$ definimos a seguinte aplicação linear $e: L(Y, X) \longrightarrow E$ tal que $T \longmapsto e(T)=\overline{\left(P_{Y Z}(T)\right)_{Z}}$, onde $Z$ é subespaço de $Y$. Observando que $E$ é completo e todo elemento não nulo de $E$ é invertível em relação ao produto definido em 1.9.3.1 (veja [15], pág. 75 e 77).

Proposição 1.9.3.4 Seja X um espaço de Banach complexo HI. Então o espaço E com a multiplicação dada na definição 1.9.3.1 é uma álgebra de Banach com unidade.

Prova : Sugerimos [15], pág.79.

Proposição 1.9.3.5 Sejam $X$ um espaço de Banach $H I$ e $Y$ um subespaço qualquer de $X$ com dimensão infinita. Então o espaço $E_{Y}$ é isométrico a um subespaço de E.

Prova : Sugerimos [15], pág.79. 
Proposição 1.9.3.6 Seja X um espaço de Banach complexo H.I.. Então, o espaço é dado na Definição 1.9.2.5 é isométrico à $\mathbb{C}$.

Prova : Sugerimos [15], pág. 79.

Corolário 1.9.3.7 Seja X um espaço de Banach complexo H.I.. Então, E possui dimensão 1.

Prova : Sugerimos [15], pág. 79. 


\section{QUASI-MAXIMALIDADE DE SUBESPAÇOS}

Neste capítulo introduziremos o conceito de subespaços quasi-maximais para em seguida demonstrarmos o Lema 2.2.1 sobre soma direta e subespaços $I d+K$-isomorfos, de grande importância na prova do Lema 3.2.2, que por sua vez auxiliará na demonstração do Teorema Principal (Teorema 7.1.1). Terminaremos este capítulo com as demonstrações dos Lemas 2.4 .1 e 2.4.2 sobre as relações da quasi-maximalidade de subespaços e operadores estritamente singulares, que desempenham papel importante nas demonstrações do Teorema 12 (ii) e do Teorema 13 que foram enunciados na Introdução.

\subsection{Quasi-maximalidade}

Definição 2.1.1 Sejam $X$ um espaço de Banach e $Y$ um subespaço de $X$. $Y$ é dito quasimaximal se para todo subespaço $Z$ de $X$, a soma $Y+Z$ não é direta.

\subsection{Soma não direta e subespaços $I d+k$-isomorfos}

Lema 2.2.1 Sejam $X$ um espaço de Banach, $Y$ e $Z$ subespaços de $X$. Se para todo subespaço $W$ de $Z$, a soma $Y+W$ não é direta, então $Y$ e $Z$ têm subespaços $I d+K$-ismorfos.

Prova : Sejam $Y$ e $Z$ subespaços de $X$ satisfazendo as hipóteses do lema. Pela Observação 1.3.3 podemos assumir que $Z$ tem uma base de Schauder $\left(e_{i}\right)_{i \in \mathbb{N}}$, com projeções $\left(p_{n}\right)_{n \in \mathbb{N}}$.

Começamos provando a seguinte afirmação: " $\forall \mathcal{E}>0$ e para algum subespaço $W$ de $Z$, se a soma $Y+W$ não é direta, então existem vetores unitários, $y \in Y$ e $w \in W$ tais que $\|y-w\| \leq \mathcal{E} "$.

Supondo que não, ou seja, existem $\mathcal{E}>0$ e $y \in Y$ e $w \in W$, vetores unitários tais que $\|y-w\|>\mathcal{E}$ temos que :

1. $Y \cap W=\{\sigma\}$. De fato, se $Y \cap W \neq\{\sigma\}$ então existe $x \neq \sigma, x \in Y \cap W$ e $0=\left\|\frac{x}{\|x\|}-\frac{x}{\|x\|}\right\|>\mathcal{E}$, o que contraria a hipótese; 
2. Pela Definição 1.7 .5 podemos concluir que : $\gamma(Y, W)=\inf \{\|y-w\|:\|y\|=\|w\|=1, y \in Y$ e $w \in W\}>\mathcal{E}>0 ;$

3. Pelo Lema 1.7.6 temos que $(Y+W)$ é fechado;

4. Seja $p_{Y}: Y+W \longrightarrow Y$, a projeção definida por $p_{Y}(y+w)=y$. Se $y_{n}+w_{n} \stackrel{Y+W}{\longrightarrow} \sigma \mathrm{e}$ $p_{Y}\left(y_{n}+w_{n}\right) \stackrel{Y}{\longrightarrow} y$, então $\sigma \in D_{p_{Y}}$ e $w_{n} \longrightarrow-y$. Logo, como $W$ é fechado temos que $-y \in Y \cap W$, e assim $y=\sigma$. Portanto, pela Observação 1.2.15.(i) e pelo Teorema do Gráfico Fechado (1.2.18), $p_{Y}$ é fechado e consequentemente $p_{Y}$ é contínuo.

5. Segue, pela Definição 1.2 .12 , que $(Y+W)$ é a soma direta o que é uma contradição. Desse modo $\|y-w\| \leq \mathcal{E}$, isto é, a afirmação é verdadeira.

Agora provaremos que é possível escolher uma base de bloco normalizada $\left(z_{i}\right)_{i \in \mathbb{N}}$ em $Z$ e uma sequência $\left(y_{i}\right)_{i \in \mathbb{N}}$ em $Y$ tal que $\sum_{i \in \mathbb{N}}\left\|z_{i}-y_{i}\right\| \leq 1$.

De fato, pela afirmação anterior existem $y_{1}^{\prime} \in Y$ e $z_{1}^{\prime} \in Z$ com $\left\|y_{1}^{\prime}\right\|=1,\left\|z_{1}^{\prime}\right\|=1$ e $\left\|z_{1}^{\prime}-y_{1}^{\prime}\right\| \leq \frac{1}{8}$. Como $\left(e_{i}\right)_{i \in \mathbb{N}}$ é base de $Z$, temos que existe uma sequência de escalares $\left(a_{i}^{1}\right)_{i \in \mathbb{N}}$ tais que $z_{1}^{\prime}=\sum_{i=1}^{\infty} a_{i}^{1} e_{i}$.

Seja $n_{1} \in \mathbb{N}$ tal que : $(a)\left\|\sum_{i=n_{1}+1}^{\infty} a_{i}^{1} e_{i}\right\| \leq \frac{1}{8} \quad$ e $\quad(b)\left\|\sum_{i=1}^{n_{1}} a_{i}^{1} e_{i}\right\| \geq \frac{1}{2}$

$\operatorname{Sejam} \overline{z_{1}}=\sum_{i=1}^{n_{1}} a_{i}^{1} e_{i}, z_{1}=\frac{\overline{z_{1}}}{\left\|\overline{z_{1}}\right\|}$ e $y_{1}=\frac{y_{1}^{\prime}}{\left\|\overline{z_{1}}\right\|}$. Logo $\left\|z_{1}\right\|=1 \mathrm{e}\left\|z_{1}-y_{1}\right\|=\left\|\frac{\overline{z_{1}}}{\left\|\overline{z \bar{z}_{1}}\right\|}-\frac{y_{1}^{\prime}}{\left\|\overline{z \bar{z}_{1}}\right\|}\right\|=$ $\frac{1}{\left\|\overline{z_{1}}\right\|} \cdot\left\|\overline{z_{1}}-y_{1}^{\prime}\right\| \stackrel{(b)}{\leq} 2 \cdot\left\|\overline{z_{1}}-y_{1}^{\prime}\right\|=2 \cdot\left\|\overline{z_{1}}-z_{1}^{\prime}+z_{1}^{\prime}-y_{1}^{\prime}\right\| \leq 2 \cdot\left[\left\|\overline{z_{1}}-z_{1}^{\prime}\right\|+\left\|z_{1}^{\prime}-y_{1}^{\prime}\right\|\right] \stackrel{(a)}{\leq} 2 \cdot\left(\frac{1}{8}+\frac{1}{8}\right)=\frac{1}{2}$.

Seja $Z_{2}=\left\{\left(\sum_{i=n_{1}+1}^{\infty} a_{i} e_{i}\right) \in Z\right\}$. Então pelo Lema 1.3.12 $Z$ é de dimensão infinita. Logo pela hipótese $Z_{2}+Y$ não é soma direta e pela afirmação inicial existem $y_{2}^{\prime} \in Y$ e $z_{2}^{\prime} \in Z$ com $\left\|y_{2}^{\prime}\right\|=1,\left\|z_{2}^{\prime}\right\|=1$ e $\left\|z_{2}^{\prime}-y_{2}^{\prime}\right\| \leq \frac{1}{16}$, sendo $z_{2}^{\prime}=\sum_{i=n_{1}+1}^{\infty} a_{i}^{2} e_{i}$ para alguma sequência de escalares $\left(a_{i}^{2}\right)_{i \in \mathbb{N}}$.

Seja $n_{2} \in \mathbb{N}$ tal que : $\quad(a)\left\|\sum_{i=n_{2}+1}^{\infty} a_{i}^{2} e_{i}\right\| \leq \frac{1}{16} \quad$ e $\quad(b) \| \sum_{i=n_{1}+1}^{n_{2}} \geq \frac{1}{2}$. 
Sejam $\overline{z_{2}}=\sum_{i=1}^{n_{2}} a_{i}^{2} e_{i}, z_{2}=\frac{\overline{z_{2}}}{\left\|\overline{z_{2}}\right\|}$ e $y_{2}=\frac{y_{2}^{\prime}}{\left\|\overline{z_{2}}\right\|}$. Logo $\left\|z_{2}\right\|=1$ e $\left\|z_{2}-y_{2}\right\|-\left\|\frac{\overline{z_{2}}}{\left\|\overline{z_{2}}\right\|}-\frac{y_{2}^{\prime}}{\left\|\overline{z_{2}}\right\|}\right\|=$
$\frac{1}{\left\|\overline{z_{2}}\right\|} \cdot\left\|\overline{z_{2}}-y_{2}^{\prime}\right\| \leq 2 \cdot\left\|\overline{z_{2}}-y_{2}^{\prime}\right\|=2 \cdot\left\|\overline{y_{2}}-z_{2}^{\prime}+z_{2}^{\prime}-y_{2}^{\prime}\right\| \leq 2 \cdot\left[\left\|\overline{z_{2}}-z_{2}^{\prime}\right\|+\left\|z_{2}^{\prime}-y_{2}^{\prime}\right\|\right] \leq 2 \cdot\left(\frac{1}{16}+\frac{1}{16}\right)=\frac{1}{4}$.

Portanto, como anteriormente, temos que $\left\|z_{2}-y_{2}\right\| \leq \frac{1}{4}$. Consequentemente, por indução, existe uma base de bloco normalizada $\left(z_{i}\right)_{i \in \mathbb{N}}$ em $Z$ e uma sequência $\left(y_{i}\right)_{i \in \mathbb{N}}$ em $Y$ tal que $\sum_{i \in \mathbb{N}}\left\|z_{i}-y_{i}\right\| \leq 1$.

Seja agora $Z^{\prime}$ o subespaço de $Z$ gerado por $\left(z_{i}\right)_{i \in \mathbb{N}}$. Como $\left(z_{i}\right)_{i \in \mathbb{N}}$ é uma base de bloco normalizada de $\left(e_{i}\right)_{i \in \mathbb{N}}$ segue pela Proposição 1.3 .15 que $\left(z_{i}\right)_{i \in \mathbb{N}}$ é base de Schauder de $Z^{\prime}$.

Definimos então o operador $A: Z^{\prime} \longrightarrow Y$ tal que $A\left(\sum_{i=1}^{\infty} a_{i} z_{i}\right)=\sum_{i=1}^{\infty} a_{i} y_{i}$. Notemos que $A$ está bem definido, pois sendo $C$ a constante básica de $\left(z_{i}\right)_{i \in \mathbb{N}}$ pela Proposição 1.3 .10 segue que a série $\sum_{i=1}^{\infty} a_{i}\left(y_{i}-z_{i}\right)$ converge. De fato, mostraremos abaixo que tal série é de Cauchy.

$\left\|\sum_{i=n+1}^{n+p} a_{i} y_{i}\right\|=\left\|\sum_{i=n+1}^{n+p} a_{i}\left(y_{i}-z_{i}\right)+\sum_{i=n+1}^{n+p} a_{i} z_{i}\right\| \leq$

$\leq 2 C\left\|\sum_{i=1}^{\infty} a_{i} z_{i}\right\|\left(\sum_{i=n+1}^{n+p}\left\|y_{i}-z_{i}\right\|\right)+\sum_{i=n+1}^{n+p}\left\|a_{i} z_{i}\right\| \leq$

$\leq 2 C\left\|\sum_{i=1}^{\infty} a_{i} z_{i}\right\|\left(\sum_{i=n+1}^{n+p}\left\|y_{i}-z_{i}\right\|\right)+(p-1) 2 C\left\|\sum_{i \in \mathbb{N}} a_{i} z_{i}\right\| \leq$

$\leq 2 C\left\|\sum_{i=1}^{\infty} a_{i} z_{i}\right\|(p-1)+(p-1) 2 C\left\|\sum_{i=1}^{\infty} a_{i} z_{i}\right\|=4 C(p-1)\left\|\sum_{i \in \mathbb{N}} a_{i} z_{i}\right\|$

Mais ainda, definimos $K: Z^{\prime} \longrightarrow X$ sendo $K\left(\sum_{i=1}^{\infty} a_{i} z_{i}\right)=\sum_{i=1}^{\infty} a_{i}\left(z_{i}-y_{i}\right)$. Como anteriormente, temos que $K$ está bem definido. E também para cada $n \in \mathbb{N}$, definimos $K_{n}: Z^{\prime} \longrightarrow X$ de modo que $K_{n}\left(\sum_{i=1}^{\infty} a_{i} z_{i}\right)=\sum_{i=1}^{n} a_{i}\left(z_{i}-y_{i}\right)$. Sendo $K_{n}$ operadores com imagens de dimensão finita segue pela Proposição 1.5.2.3 que eles são compactos.

Notemos que $K_{n}$ converge uniformemente para $K$.

De fato, seja $z=\sum_{i=1}^{\infty} a_{i} z_{i} \in Z^{\prime}$ com $\|z\|=1$. Logo pela Proposição 1.3 .10 temos que $\left|a_{i}\right| \leq 2 C$, onde $C$ é constate básica de $\left(z_{i}\right)_{i \in \mathbb{N}}$. Então dado $\mathcal{E}>0$ existe $n_{0}$ tal que para $n \geq n_{0}$ temos $\sum_{i=n+1}^{\infty}\left\|z_{i}-y_{i}\right\| \leq \frac{\mathcal{E}}{2 C}$. Portanto, para $n \geq n_{0}$ segue que $\left\|K_{n}(z)-K(z)\right\|=$ $\left\|\sum_{i=n+1}^{\infty} a_{i}\left(z_{i}-y_{i}\right)\right\| \leq 2 C \sum_{i=n+1}^{\infty}\left\|z_{i}-y_{i}\right\| \leq \mathcal{E}$. 
Sendo $\left(K_{n}\right)_{n \in \mathbb{N}}$ uma sequência de operadores compactos, pela Proposição 1.5.2.5, concluimos que $K$ é compacto.

Finalmente observe que $A=I d+K$, onde $I d: Z^{\prime} \longrightarrow Z^{\prime}$ é o operador identidade.

Em particular, pela Proposição 1.5.2.6 $A$ é da forma $I d+S$, onde $S$ é um operador estritamente singular. Logo a Proposição 1.6.1 implica que $A$ é um isomorfismo sobre a imagem do operador restrito a um subespaço $Z^{\prime \prime}$ de $Z^{\prime}$ de codimensão finita. Portanto $Z^{\prime \prime}$ e $A\left(Z^{\prime \prime}\right)$ são $I d+K$-isomorfos.

Lema 2.2.2 Sejam E e $Z$ subespaços de um espaço de Banach $X$ tais que $E$ e $Z$ têm subespaços $(I d+S)$-isomorfos. Então $E+Z$ não é uma soma direta.

Prova : Seja a aplicação $(e, z) \in(E \times Z) \longmapsto(e-z) \in(E+Z)$ tal que $\|(e, z)\|_{1}=\|e\|+\|z\|$. (1)

Suponhamos, por absurdo que $E+Z$ seja uma soma direta.

Logo, exitem constantes não nulas $c_{1}$ e $c_{2}$ tais que $c_{1}\|(e, z)\|_{1} \leq\|e-z\| \leq c_{2}\|(e, z)\|_{1}$, $\forall e \in E$ e $\forall z \in Z \quad$ (veja [15], pág.12, prova do Lema 1.7.6).

Sejam $E^{\prime}$ e $Z^{\prime}$ subespaços respectivamente de $E$ e $Z$ tais que existe um operador singular $S, \operatorname{com}(I d+S): E^{\prime} \longrightarrow Z^{\prime}$ sendo um isomorfismo sobre a imagem em $Z^{\prime}$.

Em particular existem constante não nulas $c_{3}$ e $c_{4}$ tais que $c_{3}\left\|e^{\prime}\right\| \leq\left\|(I d+S)\left(e^{\prime}\right)\right\| \leq c_{4}\left\|e^{\prime}\right\|, \forall e^{\prime} \in E^{\prime}$.

Como $S$ é estritamente singular e tomando $\mathcal{E}=\left(1+c_{3}\right) \cdot \frac{c_{1}}{2}$, temos pela Proposição 1.5.2.4, que existe um subespaço de dimensão infinita $F$ em $E^{\prime}$ tal que $\left\|\left.S\right|_{F}\right\| \leq \mathcal{E}, \forall \mathcal{E}>0$. (4)

Logo de (2) em (4) temos que para $f \in F \operatorname{com}\|f\|=1$

$$
c_{1}\|(f,(I d+S)(f))\| \leq\|f-(I d+S)(f)\|=\|S(f)\| \leq \mathcal{E} .
$$


Então, aplicando (1) na desigualdade acima temos

$$
c_{1}(\|f\|+\|(I d+S)(f)\|) \leq \mathcal{E}
$$

E tomando $\mathcal{E}=\left(1+c_{3}\right) \frac{c_{1}}{2}$ e aplicando (3) na desigualdade anterior temos

$$
c_{1}\left(1+c_{3}\right) \leq c_{1}(\|f\|+\|(I d+S)(f)\|) \leq \mathcal{E}=\left(1+c_{3}\right) \frac{c_{1}}{2}, \text { absurdo }
$$

Portanto, $E+Z$ não é uma soma direta.

\subsection{Subespaços quasi-maximais e subespaços $I d+K$-isomorfos}

Nesta seção obtemos dos Lemas 2.2.1 e 2.2.2 o Corolário 2.3.1 que será aplicado nas demonstrações dos próximos Lemas 2.4 .1 e 2.4.2.

Corolário 2.3.1 Sejam $X$ um espaço de Banach e $E$ um subespaço de $X$. Então E é quasi-maximal se, e somente se, para qualquer subespaço $Z$ de $X, Z$ e $E$ têm subespaços $(I d+S)$-isomorfos.

Prova : Seja $Z$ um subespaço qualquer de $X$. Consequentemente, se $E$ é um subespaço quasi-maximal de $X$, então pela Definição 2.1.1, para qualquer outro subespaço $Z$ de $X$, a soma $E+Z$ não é direta. Dessa maneira, pelo Lema 2.2.1 $E$ e $Z$ têm subespaços $(I d+K)$ isomorfos.

Mas pela Proposição 1.5.2.6 todo operador compacto é estritamente singular, logo temos que $E$ e $Z$ têm subespaços $(I d+S)$-isomorfos.

Reciprocamente, se para qualquer subespaço $Z$ de $X, Z$ e $E$ têm subespaços $(I d+S)$-isomorfos, então pelo Lema 2.2.2 $E+Z$ não é soma direta. Desse modo concluímos, pela Definição 2.1.1 que $E$ é um subespaço quasi-maximal de $X$.

Observação 2.3.2 O Corolário 2.3.1 também é verdadeiro se substituírmos $(I d+S)$ por 
$(I d+K)$, pois todo operador compacto é também um operador estritamente singular (Proposição 1.5.2.6).

\subsection{Quasi-maximalidade de subespaços e operadores estritamente singulares}

Nesta seção demonstraremos os importantes Lemas 2.4 .1 e 2.4.2 que serão utilizados nas provas dos Teoremas 12 (ii) e 13 que foram enunciados na Introdução.

Lema 2.4.1 Sejam $X$ um espaço de Banach e E um subespaço quasi-maximal em $X$. Sejam $Z$ um espaço de Banach e $T \in L(X, Z)$. Então $T$ é estritamente singular se, e somente se, a restrição $T_{\mid E}$ de $T$ para $E$ é estritamente singular.

Prova : Por um lado se $T$ é estritamente singular então é imediato pela própria definição que $T_{\left.\right|_{E}}$ também o seja.

Por outro lado, suponha $T_{\mid E}$ um operador estritamente singular.

Seja $Y$ um subespaço de $X$. Então pelo Corolário 2.3.1, se existe um subespaço $Y^{\prime}$ de $Y$ e um isomorfismo $A=I d_{\mid Y^{\prime}}+S$ de $Y^{\prime}$ para $E$, isso implica que :

$$
T_{\mid E} A=T_{\mid E}\left(I d_{\left.\right|_{Y^{\prime}}}+S\right)
$$

Segue de (1) que, $T_{\mid Y^{\prime}}=T\left(I d_{\mid Y^{\prime}}+S-S\right)=T_{\mid E} A-T S$.

Como $T_{\mid E}$ e $S$ são estritamente singulares, segue pela Proposição 1.5.1.6 que $T_{\mid E} A$ e TS também são estritamente singulares. Logo, pelo Corolário 1.5.1.5, $\left.T\right|_{Y^{\prime}}$ também é estritamente singular.

Em particular, pela Proposição 1.5.1.4 isso equivale a dizer que $\forall \mathcal{E}>0, \exists y^{\prime} \in Y^{\prime}$, $\left\|y^{\prime}\right\|=1$ tal que $\left\|T\left(y^{\prime}\right)\right\| \leq \mathcal{E}$. Portanto, como $Y$ é arbitrário temos pela Proposição 1.5.1.3 que $T$ é estritamente singular. 
Lema 2.4.2 Sejam $X$ um espaço de Banach e E um subespaço quasi-maximal de $X$. Sejam $Z$ um espaço de Banach e $T \in L(Z, X)$. Então, existem $Z^{\prime} \subset Z$ e $T^{\prime} \in L\left(Z^{\prime}, X\right)$ com Im $T^{\prime} \subset E$ tal que $\left(T_{\mid Z^{\prime}}-T^{\prime}\right)$ é estritamente singular.

Prova : Seja $T \in L(Z, X)$. Então temos duas possibilidades para $T$.

Se $T$ é estritamente singular, então basta $Z^{\prime}=Z$ e $T^{\prime}=0$ para que $\operatorname{Im} T^{\prime} \subset E$ e que $T_{\mid Z^{\prime}}-T^{\prime}=T_{\mid Z}-0=T_{Z}$, que é estritamente singular pelo Lema 2.4.1.

Caso contrário, $T$ não é estritamente singular, ou seja, existe um subespaço de $Z$ tal que $T$ é um isomorfismo desse subespaço sobre a sua imagem que está em $X$.

Portanto pelo Corolário 2.3.1 aplicado à $T(Z)$ e $E$, existe um subespaço $Z^{\prime}$ de $Z$ tal que através de um isomorfismo da forma $A=(I d+S), T\left(Z^{\prime}\right)$ é isomorfo a um subespaço de $E$.

Seja $T^{\prime}=A T_{\mid Z^{\prime}}$. Então, $T^{\prime}=(I d+S) T_{\mid Z^{\prime}}=T_{\mid Z^{\prime}}+S T_{\mid Z^{\prime}}$ onde tanto $T_{\mid Z^{\prime}}$ como $S T_{\mid Z^{\prime}}$ estão contidos em $E$. Logo, $\operatorname{Im} T^{\prime} \subset E$.

Além disso, pela Proposição 1.5.1.6 e 1.5.1.5 temos que $S T_{\mid Z^{\prime}}$ é estritamente singular e consequentemente $S T_{\mid Z^{\prime}}=\left(T_{\mid Z^{\prime}}-T\right)$ também o é.

Observação 2.4.3 O Lema 2.4.2 também é válido se substituírmos no enunciado operador estritamente singular por operador compacto, pois pela Proposição 1.5.2.7 segue que para todo operador $S$ estritamente singular sobre $Z$, existe um subespaço $W$ de $Z$ tal que $S_{\mid w}$ é compacto. 


\section{ESPAÇOS DE BANACH $H D_{n}$}

Neste capítulo apresentaremos o espaço de Banach $H D_{n}$ e seus primeiros resultados que serão úteis nos outros capítulos. Consequentemente, estaremos em condições de provar o primeiro resultado (Teorema 3.3.1) dessa dissertação, sobre a caracterização da inexistência de sequência básica incondicional em espaços de Banach $H D_{n}$, Teorema 12 (i) enunciado na Introdução.

\subsection{Espaço de Banach hereditariamente finitamente decomponível}

Nesta seção além de definirmos os espaços de Banach $H D_{n}$, observamos algumas relações com espaços de Banach hereditariamente indecomponíveis e a quasi-maximalidade.

Definição 3.1.1 Um espaço de Banach $X$ é hereditariamente finitamente decomponível se $o$ número máximo de subespaços de dimensões infinitas de $X$ formando uma soma direta em $X$ é finito. Para um natural $n \geq 1, X$ é $H D_{n}$ se esse número de somandos é igual a $n$.

Observações 3.1.2 Decorre da definição anterior que :

(i) um espaço de Banach é $H D_{1}$ se, e somente se, ele é H.I. (hereditariamente indecomponivel);

(ii) um espaço de Banach é $H D_{n}$ se, e somente se, contém somas diretas de $n$ subespaços e toda tal soma é quasi-maximal. Em particular, $X$ é um espaço de Banach H.I. se, e somente se, todo subespaço fechado de dimensão infinita de $X$ é quasi-maximal;

(iii) todo subespaço $Y$ fechado de dimensão infinita de um espaço de Banach $H D_{n}$ é $H D_{m}$ para algum $m \leq n$.

A seguir na próxima seção (3.2) demonstraremos os primeiros resultados em espaços de Banach $H D_{n}$. 


\subsection{Somas entre espaços $H D_{n}$}

O resultado (Proposição 3.2.2) da soma de espaços $H D_{n}$ será demonstrado utilizando o Lema 2.2.1 e a Proposição 3.2.1 que provaremos a seguir.

Proposição 3.2.1 Se $X$ é um espaço de Banach $H D_{m}$ e $s \in \mathbb{N}^{*}$, então $X \oplus \mathbb{R}^{s}$ é $H D_{m}$.

Prova : Seja $X_{1} \oplus X_{2} \oplus \cdots \oplus X_{p}$ uma soma direta em $X \oplus \mathbb{R}^{s}$. Então pela proposição 1.4 .6 $X_{i}=Y_{i} \oplus F_{i}$, onde $F_{i}$ são subespaços de dimensão finita de $X_{i}$ e $Y_{i} \subset X_{i}, \forall 1 \leq i \leq s$.

Logo, $X_{1} \oplus \cdots \oplus X_{p}=Y_{1} \oplus \cdots \oplus Y_{p} \oplus F_{1} \oplus \cdots \oplus F_{p}$ e pelas Proposições 1.2.9 e 1.2.10 $Y_{1} \oplus \cdots \oplus Y_{p}$ é uma soma direta em $X$.

Como $X$ é $H D_{m}$, temos que $p \leq m$. Consequentemente $X \oplus \mathbb{R}^{s}$ é $H D_{m}$.

Proposição 3.2.2 Sejam X um espaço de Banach $H D_{m}$ e $Y$ outro espaço de Banach $H D_{n}$. Então $X \oplus Y$ é $H D_{m+n}$.

Prova : Sejam $X$ e $Y$ espaços de Banach, respectivamente $H D_{m}$ e $H D_{n}$.

Das hipóteses temos que existem $m$ subespaços de $X$ e $n$ subespaços de $Y$ formando respectivamente uma soma direta em $X$ e outra em $Y$. Além do que, a soma de todos esses subespaços é uma soma direta de $(m+n)$ subespaços de $X \oplus Y$.

Seja o conjunto de índices $K=\{1, \ldots, k\}$ tal que $Z_{k}$ representa uma soma direta em $X \oplus Y$. Então temos que provar que $k \leq m+n$.

Sejam as projeções $p: X \oplus Y \longrightarrow X$ e $q: X \oplus Y \longrightarrow Y$. Então vamos começar provando a afirmação : "cada $\left.p\right|_{i}$ e cada $\left.q\right|_{i}$ é ou estritamente singular ou um isomorfismo em subespaço $Z_{i}$, com $1 \leq i \leq k "$.

Seja $I_{X}$ o conjunto de $i$ tal que $\left.p\right|_{i}$ é um isomorfismo, de modo que para $i \in I_{X}$ temos $X_{i}=p\left(Z_{i}\right)$. Seja $\Re$ o conjunto de subconjuntos $R$ de $I_{X}$ tal que $p$ é um isomorfismo sobre 
$Z_{R}$ ou sobre alguma soma menor (Preliminar 1.2.12).

Seja $r=\operatorname{máx}\{|R|: R \in \mathfrak{R}\}$. Como $X$ é $H D_{m}$ temos que $r \leq m$. Podemos assumir agora que $r$ está fixado para $R=[1, r]$ tal que $p_{\left.\right|_{R}}$ é um isomorfismo para uma soma menor de $Z_{i}^{\prime}$ s. Seja $p_{\mid R}^{-1}$ o isomorfismo inverso de $p_{\mid R}$.

Seja $i \in I_{X} \backslash R$. Então pela definição de $r, p$ não é um isomorfismo sobre $Z_{r} \oplus Z_{i}$, ou sobre alguma soma menor. Isso implica, em particular, que nenhum subespaço de $X_{i}$ forma uma soma direta com $X_{R}$, caso contrário, se existe $X_{i}^{\prime} \subset X_{i}$, tal que $X_{i}^{\prime}$ e $X_{R}$ formam uma soma direta, e supondo $Z_{i}^{\prime}=p^{-1}\left(X_{i}^{\prime}\right)$, então sobre $Z_{R} \oplus Z_{i}^{\prime}$, $p$ é um isomorfismo, o que seria uma contradição.

Logo pelo Lema 2.2.1 e consequentemente pela Proposição 1.5.2.6 segue que algum subespaço de $X_{i}$ é $I d+S$-isomorfo a um subespaço de $X_{R}$ onde $S$ é um operador estritamente singular. Portanto, para um subespaço $X_{i}^{\prime}$ de $Z_{i}$ temos que $X_{i}^{\prime}$ é isomorfo na imagem em $X_{R}$ por um operador da forma $I d_{\mid i}+S_{i}$.

Isso produz um isomorfismo de $Z_{i}$ na sua imagem em $Z_{R}$ da forma $b_{i}=p_{\mid R}^{-1}\left(I d_{\mid i}+S_{i}\right) p_{\mid i}$. Para $i \notin R$ e não pertecente a $I_{X}$, admitimos $b_{i}=0$.

Seja $b$ o operador de $Z_{K \backslash R}$ para $Z_{R}$ cuja matriz de ordem $(r, k-r)$ é $\left[b_{r+1}, \ldots, b_{k}\right]$.

Seja agora uma outra decomposição $Z_{K}^{\prime}=Z_{1}^{\prime} \oplus \ldots \oplus Z_{k}^{\prime}$ de $Z_{K}$, definida por $Z_{i}^{\prime}=Z_{i}$ para $1 \leq i \leq r$ e $Z_{i}^{\prime}=\left\{\left(-b_{i} z_{i}, z_{i}\right): z_{i} \in Z_{i}\right\}$ para $r+1 \leq i \leq k$. Então vamos mostrar que a restrição de $p$ para $Z_{K \backslash R}^{\prime}$ é estritamente singular.

Seja $i \in K \backslash R$. Então, ou $i \in I_{X}$ e então a restrição de $p$ para $Z_{i}^{\prime}$ é definida por $p\left(-b_{i} z_{i}, z_{i}\right)=-\left(I d+S_{i}\right) p z_{i}+p z_{i}=-S_{i} p z_{i}$, logo pela Proposição 1.5.1.6 a restrição de $p$ para $Z_{i}^{\prime}$ é estritamente singular; ou $i \notin I_{X}$ e então a restrição de $p$ para $Z_{i}^{\prime}$ é definida por $p\left(-b_{i} z_{i}, z_{i}\right)=p_{l_{i}} z_{i}$ (onde $p_{l_{i}}$ é esritamente singular).

Portanto, $p$ é estritamente singular quando restrito a $Z_{K \backslash R}^{\prime}$.

Logo, a afirmação é verdadeira, e como $q=I d-p$, segue pela Proposição 1.6 .1 que a restrição de $q$ para algum subespaço de codimensão finita de $Z_{K \backslash R}^{\prime}$ é um isomorfismo, isto é, 
existe um subespaço $W$ de codimensão finita de $Z_{K \backslash R}^{\prime}$ tal que $W \hookrightarrow Y$, ou seja $W$ é isomorfo a um subespaço de $Y$.

Considerando fixado um $W$ definido no parágrafo anterior seja agora $V \subset Z_{K \backslash R}$ tal que $Z_{K \backslash R}=W \oplus V$ e $\varphi: V \rightarrow \mathbb{R}^{s}$ um isomorfismo sobrejetor, onde $s=\operatorname{dim} V$. Então, $\left(q_{\mid W}, \varphi\right): Z_{K \backslash R} \longrightarrow Y \oplus \mathbb{R}^{s}$ definido por $(q, \varphi)(\omega, v)=(q(\omega), \varphi(v))$ é um isomorfismo sobre a imagem, isto é $Z_{K \backslash R}=W \oplus V \hookrightarrow Y \oplus \mathbb{R}^{s}$.

Como $Y$ é $H D_{n}$ segue pela Proposição 3.2 .1 que $Y \oplus \mathbb{R}^{s}$ também é $H D_{n}$. Portanto, $k-r \leq n$, ou melhor $k=k-r+r \leq n+r \leq n+m$.

Corolário 3.2.3 Sejam $X_{i}$ 's espaços de Banach H.I. para os naturais $i=1, \ldots, n$. Então o espaço $\bigoplus_{i=1}^{n} X_{i}$ é $H D_{n}$.

Prova : Da hipótese temos que cada espaço $X_{i}$ é H.I.. Segue da Observação 3.1.2.(i) que um espaço H.I. é um espaço $H D_{1}$. Consequentemente, como $\bigoplus_{i=1}^{n} X_{i}=X_{1} \oplus \cdots \oplus X_{n}$ é uma soma direta de espaços H.I. , temos que $\bigoplus_{i=1}^{n} X_{i}$ é uma soma direta de espaços $H D_{1}$.

Logo, aplicando a Proposição 3.2.2 temos que $\bigoplus_{i=1}^{n} X_{i}$ é um espaço $H D_{n}$.

O Corolário 3.2 .3 acima nos proporciona o primeira exemplo de espaços $H D_{n}$ para $n \geq 2$. Contudo, a soma direta de $n$ espaços H.I. não é o único exemplo de espaços $H D_{n}$. Um espaço $H D_{n}$ não dessa forma foi construído em [5] por uma diferente resolução.

Dois resultados que já eram conhecidos para espaços H.I., também são verdadeiros para espaços $H D_{n}$. Vamos demonstrá-los na próxima seção (3.3).

\subsection{Inexistência de base incondicional em espaços de Banach $H D_{n}$}

Já estamos em condições de provar o Teorema 3.3.1 que foi enunciado como Teorema 12 (i) na Introdução . 
Teorema 3.3.1 Todo espaço de Banach hereditariamente finitamente decomponível não contém nenhuma sequência básica incondicional.

Prova: Seja $X$ um espaço de Banach $H D_{m}$. Por absurdo, vamos supor que $X$ contém uma sequência básica incondicional $\left(e_{k}\right)_{k \in \mathbb{N}}$. Então, para todo natural fixado $n \geq 1, X$ contém a soma direta $\bigoplus_{j=1}^{n} E_{j}^{n}$, onde $E_{j}^{n}$ é o espaço definido por $\left(e_{n i+j}\right)_{i \in \mathbb{N}}$.

Logo, como $\mathbb{N}$ é infinito, podemos obter um natural $n=m+1$ que determina uma soma direta de $(m+1)$ espaços para $X$. Consequentemente, descaracteriza $X$ como $H D_{m}$, o que é uma contradição.

Proposição 3.3.2 Seja $X$ um espaço $H D_{n}$. Se $X^{k} \sim X^{l}$ então $k=l$.

Prova : Pela proposição 3.2 .2 se $X$ é $H D_{n}$ então $X^{k}$ é $H D_{n k}$ enquanto $X^{l}$ é $H D_{n l}$.

Como ambos são isomorfos, temos $n k=n l$, e assim $k=l$. 


\section{ESPAÇOS DE BANACH $H D_{n}$ FUNDAMENTAIS}

Um tipo particular de espaços $H D_{n}$ chamados espaços de Banach $H D_{n}$ fundamentais será apresentado neste capítulo, que também tem por proposito demonstrar o Lema 4.3.2 sobre operadores em espaços $H D_{n}$ fundamentais. Esse Lema será de grande importância para provarmos dois dos principais resultados desta dissertação, ou seja, os Teoremas 5.1.2 e 5.2.1 no Capítulo 5 (Teoremas 12(ii) e 13 enunciados na Introdução).

Antes daremos na seção 4.1 a definição sobre os espaços $H D_{n}$ fundamentais, além das notações e observações que auxiliam nos resultados da seção 4.2

\subsection{Espaços $H D_{n}$ fundamentais}

Definição 4.1.1 Um espaço de Banach X é um espaço $H D_{n}$ fundamental se ele é a soma direta de $n$ espaços $H I$ que tomados dois a dois são ou isomorfos ou totalmente incomparáveis.

Lembramos que dois espaços são ditos totalmente incomparáveis se nenhum subespaço do primeiro é isomorfo a algum subespaço do segundo (Definição 1.2.13).

Notações 4.1.2 Seja $\mathcal{F}$ um espaço $H D_{n}$ fundamental da forma $\mathcal{F}=\bigoplus_{i=1}^{n} F_{i}$. Então :

(i) para $i, j$ em $\{1, \ldots, n\}$, denotamos por $i \simeq j$ se $F_{i}$ e $F_{j}$ são isomorfos;

(ii) para cada $i$, denotamos por $p_{i}$, a projeção $\mathcal{F} \rightarrow F_{i}$;

(iii) para cada $i \simeq j$, denotamos por $\alpha_{i j}$ um isomorfismo estabelecido de $F_{i}$ para $F_{j}$, e por $p_{i j}$ a imagem $\alpha_{i j} p_{i}$ de $\mathcal{F}$ para $F_{j}$;

(iv) denotamos por $\alpha(\mathcal{F})$ o conjunto das classes de equivalência de índices para a relação $\simeq$;

(v) definimos o conjunto $s(\mathcal{F})$ como a sequência finita das classes de equivalência ordenadas por cardinalidade decrescente; também definimos o número positivo $n(\mathcal{F})$ por $n(\mathcal{F})^{2}=\sum_{s \in s(F)}|s|^{2}$. 
Escrevendo esses números como funções de $\mathcal{F}$ é um abuso de notação, porque a "priori", eles dependem da escolha de decomposição de $\mathcal{F}$ como $\bigoplus_{i=1}^{n} F_{i}$. Permitimos essa notação porque veremos que $s(\mathcal{F})$ e $n(\mathcal{F})$ são fatores unicamente determinados por $\mathcal{F}$. Até então, pensaremos em $s$ e $n$ como funções de $\mathcal{F}$ sujeitas a uma decomposição particular.

Veremos que $s(\mathcal{F})$ e $n(\mathcal{F})$ caracterizam de uma maneira o espaço de operadores sobre $\mathcal{F}$. Nota-se também que $n(\mathcal{F}) \leq n$.

Observação 4.1.3 Seja $\mathcal{F}$ um espaço $H D_{n}$ fundamental. Então uma soma menor do que $\mathcal{F}$ pode sempre ser escolhida fundamental (se dois espaços não são totalmente incomparáveis, então passando para subespaços podemos assumir que eles são isomorfos; repetindo esse procedimento, finalizamos com uma soma fundamental). Escolheremos sempre tais somas menores, sem necessariamente dizê-las.

É claro que ambos, $s(\mathcal{F})$ e $n(\mathcal{F})$, são preservados quando aplicamos um isomorfismo para $\mathcal{F}$. Nota-se também que nem $s(\mathcal{F})$ nem $n(\mathcal{F})$ mudam quando passamos para uma soma menor; novamente isso é um abuso de notação, mas podemos permiti-lo aqui porque a comparação de duas somas fundamentais é por definição a comparação de duas decomposições de somas. A prova é a seguinte:

Sejam $\mathcal{F}$ uma soma fundamental e $G$ uma soma menor e para simplificar a notação, assuma que $\forall i, G_{i} \subset F_{i}$. Então se $F_{i}$ e $F_{j}$ são totalmente incomparáveis, também são os subespaços $G_{i}$ e $G_{j}$.

Se $F_{i}$ e $F_{j}$ são isomorfos, então pela propriedade da quasi-maximalidade em subespaços H.I., isto é, aplicando o Corolário 2.3.1 concluímos que $G_{i}$ e $G_{j}$ não são totalmente incomparáveis, portanto precisam ser isomorfos.

\subsection{Espaços $H D_{n}$ fundamentais, espaços $H D_{n}$ e subespaços quasi- maximais}

Esta seção é necessária para estabelecer algumas relações dos espaços $H D_{n}$ funda- 
mentais com espaços $H D_{n}$ e com subespaços quasi-maximais, o que nos permitirá utilizá-las nas demonstrações dos Teoremas 12(ii) e 13 enunciados na Introdução.

Vejamos então a relação dos espaços $H D_{n}$ fundamentais com espaços $H D_{n}$.

Lema 4.2.1 Todo espaço $H D_{n}$ contém um espaço $H D_{n}$ fundamental.

Prova: Seja $X$, um espaço de Banach $H D_{n}$. Então $X$ por definição contém uma soma direta de no máximo $n$ subespaços. Cada um desses subespaços precisam ser H.I., do contrário $X$ deverá conter uma soma direta de no mínimo $n+1$ subespaços.

Por observação anterior (4.1.3), passando para subespaços apropriados, podemos assumir que essa soma é um espaço $H D_{n}$ fundamental.

Observação 4.2.2 Se $X$ é um espaço de Banach $H D_{n}$, então todo subespaço $H D_{n}$ fundamental $\mathcal{F}$ de $X$ é quasi-maximal em $X$. Essa é uma importante observação com respeito aos Lemas 2.4.1 e 2.4.2.

\subsection{Operadores em espaços de Banach $H D_{n}$ fundamentais}

No que segue seja $X$ um espaço de Banach $H D_{n}$. Estudaremos agora a posição relativa de subespaços fundamentais de $X$. Fixaremos também notações que nos ajudam na prova do Lema 4.3.2.

Notação 4.3.1 Sejam $\mathcal{F}=\bigoplus_{i=1}^{n} F_{i}$ um espaço fundamental e $\mathcal{G}=\bigoplus_{i=1}^{m} G_{i}$ um subespaço $H D_{m}$ fundamental de $\mathcal{F}$. Denotamos por $I_{\mathcal{G}, \mathcal{F}}$ a injeção de $\mathcal{G}$ na imagem em $\mathcal{F}$, escrita como uma matriz $n \times m$ com coeficientes em $L\left(G_{j}, F_{i}\right)$, para $1 \leq j \leq m$ e $1 \leq i \leq n$.

Para $1 \leq i \leq n$, lembramos que $p_{i}$ denota a projeção sobre $F_{i}$, e de acordo com a notação definida em $1.2 .12 p_{i \mid j}$ é o coeficiente da i-ésima linha e j-ésima coluna da matriz $I_{G, F}$. 
Lema 4.3.2 Sejam $\mathcal{F}=\bigoplus_{i=1}^{n} F_{i}$ um espaço $H D_{n}$ fundamental e $\mathcal{G}=\bigoplus_{i=1}^{m} G_{i}$, um subespaço fundamental de $\mathcal{F}$. Então existe uma soma $\mathcal{H}$ menor do que $\mathcal{G}$, um isomorfismo $L$ de $\mathcal{H}$ para $\mathcal{G}$ e uma permutação matricial $E$ em $\mathcal{F}$ tal que $E I_{\mathcal{G}, \mathcal{F}} L=\left(\begin{array}{c}D+S \\ V\end{array}\right)$ onde $D$ representa a matriz diagonal de bloco $(m, m)$ de um isomorfismo, $S$ a matriz $(m, m)$ de um operador estritamente singular e $V$ alguma matriz $(n-m, m)$.

Prova: Seja $H$ uma soma menor de um subespaço fundamental $G$ do espaço de Banach $H D_{n}$ fundamental $F$, tal como descritos nas hipóteses.

Vamos primeiro definir que a matriz de um operador de $H$ para $F$ é da forma Gaussiana sobre as $k$ primeiras linhas se ela é da forma $\left(\begin{array}{ccc}D+S & S^{\prime} \\ V & V^{\prime}\end{array}\right)$, onde $D$ é a matriz diagonal de bloco $(k, k)$ de um isomorfismo, $S$ (respectivamente $S^{\prime}$ ) a matriz $(k, k)$ (respectivamente $(k, m-k))$ de um operador estritamente singular, e $V$ (respectivamente $V^{\prime}$ ) alguma matriz $(n-k, k)$ (respectivamente $(n-k, m-k))$.

Seja $A_{k-1}$ uma matriz da forma Gaussiana sobre as $(k-1)$ primeiras linhas e também a matriz de um isomorfismo. Então existem uma soma $H_{k-1}$ menor do que $G_{k-1}$, um isomorfismo $L_{k-1}: H_{k-1} \longrightarrow G$, e uma permutação $E_{k-1}$ sobre $F$ tal que $E_{k-1} \cdot I_{G, F} \cdot L_{k-1}=$ $A_{k-1}$.

Seja um automorfismo $B$ sobre $H_{k-1}$. Então, $E \cdot A_{k-1} \cdot B=E \cdot\left(E_{k-1} \cdot I_{G, F} \cdot L_{k-1}\right) \cdot B=\left(E \cdot E_{k-1}\right) \cdot I_{G, F} \cdot\left(L_{k-1} \cdot B\right)=E_{k} \cdot I_{G, F} \cdot L_{k}=A_{k}$ onde $E$ é uma permutação sobre $F_{i}^{\prime} s$ e $L_{K}$ é um automorfismo de $H_{k}$ em $G$. Logo, segue que $A_{k}$ é da forma Gaussiana sobre as $k$ primeiras linhas e representa um isomorfismo.

Repetindo esse procedimento até $k=m$, obtemos o resultado esperado. Portanto precisamos determinar $B$ tal que $A_{k-1} \cdot B=A_{k}$.

Sejam $N=\{1, \cdots, n\}$ e $M=\{1, \cdots, m\}$. Então pelo fato de $A_{k-1}$ ser um isomorfismo, temos que a restrição de $A_{k-1}$ para $H_{k}$ não é estritamente singular. Logo existe $i$ tal que a restrição $p_{i \mid k}$ (Notação 4.3.1) de $H_{k}$ para $p_{i}\left(H_{k}\right)$ não é estritamente singular. Pela nossa hipótese sobre a forma Gaussiana de $A_{k-1}$, temos que $i \geq k$. Consequentemente também é possível afirmar que a menos de uma permutação sobre os $F_{i}$ 's, podemos assumir que $i=k$. 
Passando para uma soma menor, podemos assumir que para cada $j$ a restrição $p_{k \mid j}$ de $H_{j}$ para $p_{k}\left(H_{j}\right)$ é estritamente singular ou um isomorfismo.

Seja $J$ o conjunto de $j$ tais que $p_{k \mid j}$ é um isomorfismo. Em particular, $k \in J$. Seja $C_{j}=p_{k}\left(H_{j}\right)$, para cada $j$ em $J$. Então $C_{j}$ e $C_{k}$ são subespaços de dimensões infinitas do espaço hereditariamente indecomponível $F_{k}$. Logo, aplicando o corolário 2.3.1, $C_{j}$ e $C_{k}$ têm subespaços $(I d+S)$-isomorfos passando para uma soma menor, podemos assumir que $C_{k}=\left(I d+s_{j}\right)\left(C_{j}\right)$. Em particular, escolhemos $s_{k}=0$.

Seja $b_{j}$ o operador $p_{k \mid k}^{-1}\left(I d_{\mid j}+s_{j}\right) p_{k \mid j}$ de $H_{j}$ para $H_{k}$ com $j \in J$, ou $b_{j}=0$ para $j \notin J$. Então a matriz $\left[b_{1}, \cdots, b_{m}\right]$ é matriz do operador $b$ de $H_{M}$ para $H_{k}$.

Desse modo, $B$ é o automorfismo sobre $H_{M}$ com matriz $\left(\begin{array}{ccc}I d_{k-1} & 0 & 0 \\ -b_{\mid[1, k-1]} & I d & -b_{\mid[k+1, m]} \\ 0 & 0 & I d_{m-k}\end{array}\right)$.

Vamos agora verificar se $A_{k}=A_{k-1} \cdot B$.

Como $A_{k-1}$ é da forma $\left(\begin{array}{ccc}\operatorname{diag}\left(p_{i}\right)_{1}^{k-1}+S_{1} & S_{2} & S_{3} \\ p_{k \mid[1, k-1]} & p_{k \mid k} & p_{k \mid[k+1, m]} \\ V_{1} & V_{2} & V_{3}\end{array}\right)$ segue que $A_{k}$ é da forma
$\left(\begin{array}{ccc}\operatorname{diag}\left(p_{i}\right)_{1}^{k-1}+S_{1}^{\prime} & S_{2} & S_{3}^{\prime} \\ \left(p_{k}-p_{k} b\right)_{\mid[1, k-1]} & p_{k \mid k} & \left(p_{k}-p_{k} b\right)_{k \mid[k+1, m]} \\ V_{1}^{\prime} & V_{2} & V_{3}^{\prime}\end{array}\right)$ onde todos os coeficientes da k-ésima linha exceto $p_{k \mid k}$ são agora estritamente singulares.

Realmente, seja $j \neq k$; então ou $j \notin J$ e então $\left(p_{k}-p_{k} b\right)$ é cquivalente a $p_{\mid j}$ estritamente singular, ou $j \in J$ e então $\left(p_{k}-p_{k} b\right)_{\mid j}=p_{k \mid j}-\left(I d_{\mid j}+s_{j}\right) p_{k \mid j}=-s_{j} p_{k \mid j}$, tal que é também estritamente singular.

Portanto, $A_{k}$ é da forma Gaussiana sobre as $k$ primeiras linhas, e também é a matriz de um isomorfismo. 
Corolário 4.3.3 Sejam $\mathcal{F}=\bigoplus_{i=1}^{n} F_{i}$ um espaço de Banach $H D_{n}$ fundamental $e$ $\mathcal{G}=\bigoplus_{i=1}^{m} G_{i}$ um subespaço $H D_{m}$ fundamental de $\mathcal{F}$. Então existem uma soma $\mathcal{H}$ menor do que $\mathcal{G}$, um subconjunto $M^{\prime}$ de $\{1, \ldots, n\}$ de cardinalidade $m$ e um isomorfismo $A$ sobre $\mathcal{H}$ tal que $A(\mathcal{H})$ é menor do que $F_{M^{\prime}}$.

Prova: Sejam $F$ e $G$ tal como na hipótese enunciada. Então, fixado um $M^{\prime}=\{1, \cdots, m\}$ contido em $\{1, \cdots, n\}$, e aplicando o Lema 4.3.2 determina-se que existe uma soma $H$ menor do que $G$, um automorfismo $B$ sobre $H$ e um isomorfismo $L: H \longrightarrow \sum_{i=1}^{m} F_{i}$ que compostos a menos de uma permutação sobre $F_{i}^{\prime} s$, finalizada num conjunto $M^{\prime}$ de cardinalidade $m$ determina $Z=L B(H)$ menor do que $\bigoplus_{i=1}^{m} F_{i}=F_{M^{\prime}}$.

Proposição 4.3.4 Sejam $X$ um espaço de Banach $H D_{n}$ e $\mathcal{F}$ e $\mathcal{F}^{\prime}$ subespaços $H D_{n}$ fundamentais de $X$. Então existem um $\mathcal{G}$ subespaço $H D_{n}$ fundamental de $\mathcal{F}$ e respectivamente $\mathcal{G}^{\prime}$ de $\mathcal{F}^{\prime}$ tais que $\mathcal{G}$ e $\mathcal{G}^{\prime}$ são isomorfos.

Prova : Seja $X$ um espaço de Banach $H D_{n}$. Sejam $F=\bigoplus_{i=1}^{n} F_{i}$, e $F^{\prime}=\bigoplus_{i=1}^{n} F_{i}^{\prime}$ subespaços $H D_{n}$ fundamentais de $X$.

Seja a soma $G$ menor do que $F$. Então pela Observação 3.1.2.(ii), $G$ é quasi-maximal e consequentemente aplicando Corolário 2.3.1, $G$ e $F^{\prime}$ têm subespaços $(I d+S)$-isomorfos.

Como $F^{\prime}$ é fundamental em $X$, temos pela Observação 3.1.2.(ii) que $F^{\prime}$ é quasimaximal. Logo, aplicando o Corolário 2.3.1, $\forall i \in\{1, \cdots, n\}$, achamos $G_{i}$ de $F_{i}$ que isomorfa com um subespaço de $F^{\prime}$ por um operador da forma $(I d+S)$, isto é, existe $\left(B_{i}=I d+S_{i}\right)_{\mid G_{i}}$.

Assim a soma $G=\bigoplus_{i=1}^{n} G_{i}$ que é menor do que $F$, isomorfa com uma soma em $F^{\prime}$ por um operador $B$ da forma $I d+S$, ou seja, $B: G \longrightarrow F^{\prime}$ tal que $B=\left\{B_{1}, \cdots, B_{n}\right\}$, para cada subespaço de codimensão finita de $G$, pela Proposição 1.6.1.

Seja $Z=B(G)$. Então, como $G \subset F$ podemos aplicar o Corolário 4.3.3, sem perda de generalidade, tendo uma nova restrição dos $F_{i}^{\prime} s$ que é um isomorfismo $L$ tal que $L\left(Z_{i}\right)$ é menor do que $F_{i}^{\prime}$. 
Portanto a soma $G^{\prime}=L(Z)=L B(G)$ é menor do que $F^{\prime}$ e isomorfa com $G$.

Agora somos capazes para estudar o espaço de operadores sobre um espaço complexo $H D_{n}$ fundamental, e então um espaço complexo $H D_{n}$ em geral. Isto será feito no próximo capítulo. 


\section{OPERADORES EM ESPAÇOS DE BANACH COMPLEXO $H D_{n}$}

No presente capítulo demonstraremos os Teoremas 12 (ii) e 13 mencionados na Introdução (respectivamente Teoremas 5.1 .2 e 5.2.1, neste capítulo). Ambos são sobre os espaços de operadores em espaços de Banach $H D_{n}$.

\subsection{Operadores em subespaços de um espaço de Banach complexo $H D_{n}$ fundamental}

Nesta seção 5.1 provaremos o Teorema 5.1.2, onde usaremos os Lemas 2.4.1 e 4.3.2. Além desses resultados faz-se necessário lembrarmos primeiro o Teorema 5.1.1 demonstrado em [3].

Teorema 5.1.1 Sejam $X$ um espaço de Banach complexo H.I. e $Y$ um subespaço de $X$. Então cada operador de $Y$ para $X$ é da forma $\lambda I_{Y, X}+S$, onde $\lambda \in \mathbb{C}, I_{Y, X}$ é a imagem da inclusão canônica de $Y$ em $X$, e $S$ é um operador estritamente singular.

No que segue nesta seção, seja $\mathcal{E}=\bigoplus_{i=1}^{n} E_{i}$ um espaço de Banach complexo $H D_{n}$ fundamental. Lembremos que $p_{i j}$ denota $\alpha_{i j} p_{i}$, onde $p_{i}$ é a projeção sobre $E_{i}$, e $\alpha_{i j}$ um isomorfismo dado de $E_{i}$ para $E_{j}$ (Notação 4.1.2.(iii)). Denotamos por $\sum_{i \simeq j}$ a soma $\sum_{C} \sum_{i, j \in C}$, onde $C$ percorre as classes de índices com $\simeq$ (Notação 4.1.2.(ii) e (iv)).

Teorema 5.1.2 Sejam $\mathcal{E}=\bigoplus_{i=1}^{n} E_{i}$ um espaço de Banach complexo $H D_{n}$ fundamental e $Y$ um subespaço de dimensão infinita de $\mathcal{E}$. Então todo operador de $Y$ tem a forma $\sum_{i \simeq j} \lambda_{i j} p_{i j}+S$, onde $\lambda_{i j}$ é uma constante e $S$ é um operador estritamente singular.

Prova : Sejam $Y$ satisfazendo as hipóteses e $S$ um operador estritamente singular em $Y$. . Logo, $Y$ é $H D_{m}$ com $m \leq n$. 
Seja $\mathcal{F}=\bigoplus_{i=1}^{m} F_{i}$ um subespaço $H D_{m}$ fundamental de $Y$. Então conforme o Lema 4.3.2, nós podemos, renumerando os $E_{i}^{\prime} s$, assumir que a matriz $I_{\mathcal{F}, \mathcal{E}}$ de injeção de $\mathcal{F}$ em $\mathcal{E}$ é da forma $\left(\begin{array}{c}D+S \\ V\end{array}\right)$, onde $D$ é a matriz diagonal de blocos $(m, m)$ de um isomorfismo, $S$ é a matriz $(m, m)$ de um operador estritamente singular, e $V$ é alguma matriz $(n-m, m)$.

Podemos também assumir, passando para uma soma menor, que para todo $i \in\{1, \ldots, m\}, p_{i \mid i}$ é um isomorfismo. Para todo tal $i$, seja $H_{i}=p_{i}\left(F_{i}\right)$. Seja $p_{i \mid i}^{-1}$ o operador inverso de $p_{i \mid i}$ definido sobre $H_{i}$.

Seja agora $T$ um operador de $Y$ para $\mathcal{E}$. Para cada $j \simeq i, H i$ é isomorfo por $\alpha_{i j}$ a um subespaço de $E_{j}$, portanto pelo Teorema 5.1.1, cada operador de $H i$ para $E_{j}$ é da forma $\lambda \alpha_{i j}+S$; enquanto para todo $j \neq i, H_{i}$ e $E_{j}$ são totalmente incomparáveis, portanto cada operador de $H_{i}$ para $E_{j}$ é estritamente singular.

Como o operador $T p_{i \mid i}^{-1}$ é de $H_{i}$ para $\mathcal{E}$, temos que $T p_{i \mid i}^{-1}$ é da forma $\sum_{i \simeq j} \lambda_{i j} \alpha_{i j}+S_{i}$, onde $S_{i}$ é um operador estritamente singular. Segue que para cada $i \leq m, T_{\mid F_{i}}$ é da forma $\left(\sum_{j \simeq i} \lambda_{i j} p_{i j}\right)_{\mid F_{i}}+S_{i}$. Se $\lambda_{i j}=0$ para todo $i \geq m+1$, então $T_{\mid \mathcal{Y}}=\sum_{i \simeq j} \lambda_{i j} p_{i j}+S$.

Portanto a restrição para $\mathcal{F}$ do operador $S$ definido por $S=T-\sum_{i \simeq j} \lambda_{i j} p_{i j}$ é estritamente singular. Consequentemente, pelo Lema 2.4.1, $S$ é também estritamente singular sobre $Y$.

No que segue apresentaremos na próxima seção 5.2 o Teorema 5.2.1 ou Teorema 13 como está enunciado na Introdução.

\subsection{Dimensão do espaço quociente entre operadores de subespaços de um espaço de Banach complexo $H D_{n}$ e seus operadores estrita- mente singulares}

Para a demonstração do Teorema 5.2.1, além do resultado obtido na seção anterior (Teorema 5.1.2), usaremos também os Lemas 2.4.1, 2.4.2 e 4.3.2. 
Teorema 5.2.1 Seja $X$ um espaço de Banach complexo $H D_{n}$. Então :

$\operatorname{dim}(L(X) / S(X)) \leq n(X)^{2} \leq n^{2}$.

Prova : Vejamos a afirmação abaixo.

"Sejam $X$ um espaço de Banach complexo $H D_{n}$ e $Y$ subespạ $H D_{m}$ de $X$.

Então $\operatorname{dim}(L(Y, X) / S(Y, X)) \leq n(Y) \cdot n(X) \leq m \cdot n "$.

Nota-se que provando a afirmação acima, a demonstração do Teorema é imediata, pois como por corolário basta fazermos $Y=X$.

Sejam $X$ e $Y$ como descriminados na afirmação. Sejam $\mathcal{F}=\bigoplus_{i=1}^{m} F_{i}$ um subespaço $H D_{m}$ fundamental de $Y$ e $\mathcal{E}=\bigoplus_{i=1}^{n} E_{i}$ um subespaço $H D_{n}$ fundamental de $X$ contendo $\mathcal{F}$. Sem perda de generalidade, podemos também assumir que a injeção de $\mathcal{F}$ para $\mathcal{E}$ é da forma dada pelo Lema 4.3.2.

Seja $T \in L(\mathcal{F}, X)$. Como $\mathcal{E}$ é quasi-maximal em $X$, para $1 \leq i \leq m$, podemos aplicar o Lema 2.4.2 onde $Z=F_{i}$; obtendo $F_{i}^{\prime} \subset F_{i}$ e $T_{i}^{\prime}$ uma perturbação estritamente singular de $T_{\mid F_{i}^{\prime}} \operatorname{com} \operatorname{Im} T_{i}^{\prime} \subset \mathcal{E}$.

Seja $\mathcal{F}^{\prime}=\sum_{i=1}^{m} F_{i}^{\prime}$. Então podemos assumir que $\mathcal{F}^{\prime}$ é fundamental e $T^{\prime}$ é o único operador sobre $\mathcal{F}^{\prime}$ tal que $T_{\mid F_{i}^{\prime}}^{\prime}=T_{i}^{\prime}$ toma seus valores em $\mathcal{E}$ para todo $i$ e $T^{\prime}$ é uma perturbação estritamente singular de $T_{\mid \mathcal{F}^{\prime}}$.

Logo, as mesmas formas de $T^{\prime}$ e $T_{\mid \mathcal{F}^{\prime}}$ são dadas pelo Teorema 5.1.2.

Como $\mathcal{F}^{\prime}$ é quasi-maximal em $\mathcal{F}$, temos pelo Lema 2.4.1 que $T$ é da mesma forma sobre a totalidade de $\mathcal{F}$. Agora por causa da forma de $\mathcal{F}$, os elementos de alguma classe de equivalência em $\alpha(\mathcal{F})$ são isomorfos com os elementos de uma e somente uma classe de equivalência em $\alpha(\mathcal{E})$. Isso define uma imagem da aplicação quociente e de $\alpha(\mathcal{F})$ para $\alpha(\mathcal{E})$. Segue que $\operatorname{dim}(L(\mathcal{F}, X) / S(\mathcal{F}, X))=\sum_{\alpha \in \alpha(\mathcal{F})}|\alpha| \cdot|e(\alpha)|$, portanto pela desigualdade de Cauchy-Schywarz $\operatorname{dim}(L(\mathcal{F}, X) / S(\mathcal{F}, X)) \leq n(\mathcal{F}) \cdot n(\mathcal{E})$.

Agora para $Z$ subespaço fechado de dimensão infinita de $X$ e $U \in L(Z, X)$, seja $\tilde{U}$ a classe de operadores módulo $U$ estritamente singulares. Definimos uma imagem 
$\delta: L(Y, X) / S(Y, X) \rightarrow L(\mathcal{F}, X) / S(\mathcal{F}, X)$ por $\delta(\tilde{T})=\tilde{T_{\mid \mathcal{F}}}$. É claro que $\delta$ é um a aplicação linear e pelo Lema 2.4.1 é uma injeção. Logo, segue que :

$\operatorname{dim}(L(Y, X) / S(Y, X)) \leq n(Y) n(X) \leq m \cdot n$. 


\section{TEORIA ESPECTRAL EM ESPAÇOS $H D_{n}$}

Neste capítulo estudaremos a teoria espectral em espaços $H D_{n}$ para demonstrar o Lema 6.3.1 sobre operadores semi-Fredhom e Fredholm em espaços de Banach $H D_{n}$. Esse lema irá desempenhar um papel importante na demonstração do Teorema Principal (Teorema 7.1.1).

Primeiramente relembremos algumas definições na Seção 6.1 que são necessárias para os resultados da teoria espectral em espaços $H D_{n}$ que serão demonstrados na Seção 6.2.

\subsection{Definições na teoria espectral em espaços $H D_{n}$}

Definição 6.1.1 Sejam $X$, espaço de Banach e $T \in L(X)$. Já vimos que $T$ é Fredholm se sua imagem é fechada, e seus kernel e cokernel têm dimensões finitas. Dizemos ainda que $T$ é semi-Fredholm se sua imagem é fechada, e seu kernel ou cokernel tem dimensão finita. Por fim, relembramos que o indice generalizado de Fredholm determinado por $i(T)=$ $\operatorname{dim}(\operatorname{Ker} T)-\operatorname{dim}(\operatorname{coKer} T)$, é definido e adiante provamos que é contínuo sobre o conjunto de operadores semi-Fredholm.

Denotamos agora, por $S(T)$ o espectro essencial de $T$ assim definido $\{\lambda \in \mathbb{C}: \exists(T-\lambda I d): X \longrightarrow X$, operador contínuo que não é Fredholm $\}$, e por $\delta S(T)$ a fronteira de $S(T)$.

Lembremos da Proposição 1.5.3.9 onde afirma que operadores de Fredholm $T \in L(X)$ são exatamente $[T]$ invertíveis, isto é, a classe de operadores compactos invertíveis na Álgebra de Calkin $L(X) / K(X)$ (Definição 1.5.3.8). Logo, pela definição o espectro essencial de $T$ é o espectro da classe de operadores $T$ não invertíveis na Álgebra de Calkin.

Definição 6.1.2 Sejam $X$ um espaço de Banach e $T \in L(X)$. Então, o operador $T$ é dito infinitamente singular se para cada subespaço $X^{\prime}$ de codimensão finita de $X$, a restrição de $T$ para $X^{\prime}$ năo é um isomorfismo, o que equivale a dizer pela Proposição 1.5.2.7 que para todo $\mathcal{E}>0$, existe um subespaço $Y$ de dimensão infinita de $X$ tal que $\left\|T_{\mid Y}\right\| \leq \mathcal{E}$. 
Definição 6.1.3 Um escalar $\lambda$ é infinitamente singular para um operador $T$ de $L(X)$, onde $X$ é um espaço de Banach, se $T-\lambda I d$ é infinitamente singulares. Denotamos por $I(T)$ o conjunto de valores infinitamente singulares para $T$.

Na seção a seguir serão apresentados os resultados envolvendo espectro essencial de operadores sobre espaços de Banach, que serão úteis na prova do Lema 6.3.1.

\subsection{Características do espectro essencial de um operador sobre espaços de Banach}

Inicialmente vamos demonstrar um resultado sobre continuidade no índice generalizado de Fredholm.

Proposição 6.2.1 Sejam $X$ um espaço de Banach e $T \in L(X)$. Então, o índice generalizado de Fredholm para operadores $(T-\lambda I d) \in L(X)$ denotado por $i(T-\lambda I d)$ é definido e contínuo sobre o conjunto dos operadores $(T-\lambda I d)$ semi-Fredholm, onde $\lambda \in A \subset \mathbb{C}$ tem valor discreto.

Prova : Primeiramente observe que $A=\{\lambda \in \mathbb{C} /(T-\lambda I d)$ ou é um operador Fredholm ou semi-Fredholm \}.

Seja $\varphi$ uma função sobre $A$ a valores em $\mathbb{Z} \cup\left\{{ }_{-}^{+} \infty\right\}$ definida por $\varphi(\lambda)=i(T-\lambda I d)$. Observa-se, por definição do índice generalizado de Fredholm que $(T-\lambda I d)$ ou é Fredholm ou semi-Fredholm. Para provarmos que $\varphi$ é contínua, vamos decompô-la em duas funções a saber,

$f: A \longrightarrow L(X)$ e $g: L(X) \longrightarrow \mathbb{Z} \cup\left\{{ }_{-}^{+} \infty\right\}$, definidas respectivamente por $f(\lambda)=T-\lambda I d \mathrm{e}$ $g(T-\lambda I d)=i(T-\lambda I d), \forall T \in L(X)$ tais que $\varphi=g \circ f$.

Podemos afirmar que $f$ é contínua $\forall \lambda \in A$, pois fixado um $\lambda_{0}$ arbitrariamente pertencente a $A$ e um $T \in L(X)$ temos que $\forall \mathcal{E}_{f}>0, \exists \delta_{f}>0$ tal que se $\left\|\lambda-\lambda_{0}\right\|<\delta_{f}$ então $\left\|f(\lambda)-f\left(\lambda_{0}\right)\right\|=\left\|(T-\lambda I d)-\left(T-\lambda_{0} I d\right)\right\|=\left|\lambda-\lambda_{0}\right|<\delta_{f}, \forall \lambda \in A$, portanto basta $\mathcal{E}_{f}=\delta_{f}$. 
Também podemos afirmar que $g$ é contínua $\forall F \in \operatorname{Im} f$, onde $\operatorname{Im} f=D g$, pois fixado um $T \in L(X)$ e um $F_{0}$ qualquer da $\operatorname{Imf}$ temos que $\forall \mathcal{E}_{g}>0, \exists \delta_{g}>0$ tal que se $\left\|F-F_{0}\right\|=\|H\|<\delta_{g}$ então $\left|g\left(F_{0}+H\right)-g\left(F_{0}\right)\right|=\left|i\left(F_{0}+H\right)-i\left(F_{0}\right)\right|, \forall F \in D g$.

Uma vez que (1) é verdade, segue da proposição 1.5.3.10.(iii) que $\left|i\left(F_{0}+H\right)-i\left(F_{0}\right)\right|=$ $\left|i\left(F_{0}\right)-i\left(F_{0}\right)\right|=0<\mathcal{E}_{g}$, qualquer que seja $\delta_{g}>0$.

Logo, como ambas funções são contínuas, temos que a função composta $\varphi=g \circ f$ é também contínua para todo $\lambda \in A \subset \mathbb{C}$.

Vejamos agora a Proposição 6.2.2, que junto com a proposição anterior são usadas nas demonstrações dos próximos resultados.

Proposição 6.2.2 Sejam $X$ um espaço de Banach e $T \in L(X)$. Então o espectro essencial de $T, S(T)$, é fechado.

Prova : Considerando $X$ e $T$ como nas hipóteses, vamos provar que o complemento algébrico de $S(T)$, isto é, $\mathbb{C} \backslash S(T)$ é aberto.

Como pela Proposição 6.2.1. o índice generalizado de Fredholm, definido num subconjunto $A \subset \mathbb{C}$ que caracteriza os $\lambda \in \mathbb{C}$ tais que os operadores da forma $(T-\lambda I d)$ são Fredholm ou são semi-Fredholm, apresenta-se como um função contínua, temos que $\mathbb{C} \backslash S(T)=\{\lambda \in \mathbb{C} /(T-\lambda I d)$ é Fredholm $\}$, contido em $A$ é aberto. Provemos tal afirmação.

Supondo por absurdo que $\mathbb{C} \backslash S(T)$ é fechado, seja $\lambda \in \delta(\mathbb{C} \backslash S(T))$. Então, existe $i(T-\lambda I d)$.

Por outro lado, temos que $i(T-\lambda I d)$ não está definido $\forall \lambda \in \mathbb{C} \backslash A$.

Uma vez que são verdades as afirmações acima, segue que $\forall r>0$, pela Proposição 6.2.1 existe uma bola de raio $r$ e centro em $\lambda$ contendo um conjunto $\left\{\lambda^{\prime} \in A: i\left(T-\lambda^{\prime} I d\right)=\infty\right\}$. Além disso pela continuidade da mesma Proposição 6.2.1 temos que $\forall \mathcal{E}>0$, existe $\lambda$ tal que $\forall \lambda^{\prime} \in B_{r}(\lambda)$, se $\left\|\lambda-\lambda^{\prime}\right\| \leq r$ então $\forall k \in \mathbb{N}$ temos 
$\left|i(T-\lambda I d)-i\left(T-\lambda^{\prime} I d\right)\right|=|K-\infty|<\mathcal{E}$, absurdo.

Portanto, $\mathbb{C} \backslash S(T)$ é aberto.

Provaremos agora os Lemas 6.2.3 e 6.2.4 sobre respectivamente a fronteira e a cardinalidade do espectro essencial de um operador sobre espaço de Banach que nos auxiliarão na demonstração do Lema 6.3.1

Lema 6.2.3 Sejam $X$ um espaço de Banach e $T \in L(X)$. Então, $\delta S(T) \subset I(T)$.

Prova : Sejam $X$ e $T$ tal como nas hipóteses. Então, pela definição do espectro essencial de $T, S(T)=\{\lambda \in \mathbb{C}:(T-\lambda I)$ operador contínuo que não é Fredholm $\}$, e pela Proposição 1.5.3.9 $(T-\lambda I d)$ não é invertível na álgebra de Calkin $L(X) / K(X)$.

Seja agora $\lambda \in \delta S(T)$. Então, como $S(T)$ é fechado, ou seja, $\delta S(T) \subset S(T)$, temos que $(T-\lambda I d)$ também não é Fredholm. E nem é semi-Fredholm, pois senão, pela continuidade demonstrada na Proposição 6.2.1 na função de imagem $i(T-\lambda I d)$ existiria $\lambda^{\prime}$ em uma vizinhança de $\lambda$ tal que $\left(T-\lambda^{\prime} I d\right)$ seria semi-Fredholm de índice infinito, contrariando a hipótese de $\lambda \in \delta S(T) \subset S(T)$.

Uma vez que a afirmação anteiror é verdadeira segue da Definição 6.1.1 que $\forall \lambda \in \delta S(T)$ ou $\operatorname{dim} \operatorname{Ker}(T-\lambda I d)=\infty$ ou $(T-\lambda I d)$ não é fechado.

Vamos caracterizar um subespaço de $X$ de acordo com a Definição 6.1.2.

Seja $X^{\prime}$ subespaço fechado de dimensão infinita e de codimensão finita de $X$. Então, pela Proposição 1.4.5, $X^{\prime}$ é complementado em $X$. Logo, pelas Proposições 1.2.10 e 1.4.6.(i) existe um subespaço $N$ de dimensão finita $n$ de $X$ tal que $X=X^{\prime} \ominus N$.

No caso da $\operatorname{dim} \operatorname{Ker}(T-\lambda I d)=\infty$, seja $H$ um subespaço de dimensão infinita tal que $H \subset K \operatorname{Ker}(T-\lambda I d)$. Então, $H \cap X^{\prime} \neq\{\sigma\}$.

Portanto, existe $x \in H \cap X^{\prime}$ e $x \neq \sigma$ tal que $(T-\lambda I d)(x)=\sigma$. Assim, $(T-\lambda I d)$ 
não é injetor. Logo, $(T-\lambda I d)$ não possui um isomorfismo.

Dessa maneira, $T-\lambda I d$ pelas Definições 6.1 .2 e 6.1 .3 é infinitamente singular e $\lambda \in I(T)$.

Agora, no caso em que $(T-\lambda I d)(X)$ não é fechado, considerando a mesma decomposição $X=X^{\prime} \oplus N$, temos $(T-\lambda I d)(X)=(T-\lambda I d)\left(X^{\prime}+N\right)=(T-\lambda I d)\left(X^{\prime}\right)+$ $(T-\lambda I d)(N)$.

Supondo por absurdo que $(T-\lambda I d)_{\left.\right|_{X^{\prime}}}$ é um isomorfismo temos pelas Proposições 1.2 .18 e 1.2 .14 que $(T-\lambda I d)_{\left.\right|_{X^{\prime}}}$ é fechado.

Supondo agora que para toda a sequência $\left(x_{n}\right)_{n \in \mathbb{N}}$ em $X$, com $x_{n} \longrightarrow x$ e $(T-\lambda I d)\left(x_{n}\right) \longrightarrow y \in X$, temos inicialmente que $x \in D(T-\lambda I d)_{\left.\right|_{X^{\prime}}}$, pois $X^{\prime}$ é fechado por hipótese e $N$ é fechado pela Proposição 1.2.24. Como $x_{n} \longrightarrow x$, temos que $(T-\lambda I d)\left(x-x_{n}\right) \longrightarrow \sigma, \log 0\left(T(x)-T\left(x_{n}\right)\right)-\lambda\left(x-x_{n}\right) \longrightarrow \sigma$. Dessa maneira, $T(x) \rightarrow T\left(x_{n}\right)$, portanto $T(x) \rightarrow y$.

Logo pela Proposição 1.2.15.(i) $(T-\lambda I d)(N)$ é fechado e pela Proposição 1.2.6 (T$\lambda I d)(X)$ é fechado, o que é uma contradição.

Portanto, não existe $X^{\prime}$, subespaço de $X$ tal que $T_{X_{X^{\prime}}}$ seja um isomorfismo.

Desse modo, em ambos os casos $(T-\lambda I d)$ é infinitamente singular e $\operatorname{assim} \lambda \in I(T)$.

Proposição 6.2.4 Sejam $X$ um espaço de Banach complexo $H D_{n}$ e $T \in L(X)$. Então a cardinalidade de $S(T)$ satisfaz $|S(T)| \leq n$.

Prova: Para isto é suficiente provar que $|I(T)| \leq n$. Realmente, disto logo segue pela Proposição 6.2 .3 que $|\delta S(T)| \leq n$, de modo que $S(T)$ contém no máximo $n$ pontos isolados.

Das Definição 6.1.2 e 6.1.3 temos que para cada $\lambda$ em $I(T)$ e cada $\mathcal{E}>0$ existe um subespaço $\mathcal{Y}_{\lambda}(\mathcal{E})$ de $X$, de codimensão finita sobre o qual $\left\|(T-\lambda I d)_{\mid \mathcal{Y}_{\lambda}(\mathcal{E})}\right\| \leq \mathcal{E}$. 
Paralelamente, podemos assumir que a aplicação $\mathcal{E} \longmapsto \mathcal{Y}_{\lambda}(\mathcal{E})$ é crescente, $\forall \lambda \in I(T)$, usando uma sequência básica normalizada $\left(y_{n}\right)_{n \in \mathbb{N}}$ de valores tais que $\left\|T\left(y_{n}\right)-\lambda y_{n}\right\| \leq 2^{-n}$. Senão vejamos :

Sejam $\left(y_{n}\right)_{n \in \mathbb{N}}$ uma sequência básica normalizada e escalares $\left(\alpha_{n}\right)_{n \in \mathbb{N}}$ tais que $\forall y \in \mathcal{Y}_{\lambda}\left(\mathcal{E}_{k}\right)$, com $\lambda \in I(T)$ fixado e $\mathcal{E}_{k}>0$ para um finito $\mathrm{k}$, temos $\left\|T\left(y_{n}\right)-\lambda y_{n}\right\|<2^{-n} \mathrm{e}$ $\left\|(T-\lambda I d)_{\mid y_{\lambda}\left(\varepsilon_{k}\right)}\right\|<\mathcal{E}_{k}$. Então temos que :

$\|(T-\lambda I d)(y)\| \leq\left|\alpha_{1}\right| \cdot\left\|\left(T\left(y_{1}\right)-\lambda y_{1}\right)\right\|+\cdots+\left|\alpha_{k}\right| \cdot\left\|T\left(y_{k}\right)-\lambda y_{k}\right\|+\cdots \leq\left|\alpha_{1}\right| \cdot 2^{-1}+\cdots+$ $\left|\alpha_{k}\right| \cdot 2^{-k}+\cdots \leq \max _{i \in \mathbb{N}}\left|\alpha_{i}\right|=\mathcal{E}_{k}$

Sejam $\mathcal{E}_{k+1}>\mathcal{E}_{k}>0$. Então, provamos que $\exists \mathcal{Y}\left(\mathcal{E}_{k+1}\right)$ tal que $\mathcal{Y}_{\lambda}\left(\mathcal{E}_{k}\right) \subset \mathcal{Y}_{\lambda}\left(\mathcal{E}_{k+1}\right)$ e $\left\|(T-\lambda I d)_{\left.\right|_{\gamma_{\lambda}\left(\mathcal{E}_{k+1}\right)}}\right\| \leq \mathcal{E}_{k+1}, \forall y \in \mathcal{Y}_{\lambda}\left(\mathcal{E}_{k+1}\right)$.

Se existe um $y \in \mathcal{Y}_{\lambda}\left(\mathcal{E}_{k}\right)$ tal que $y \notin \mathcal{Y}_{\lambda}\left(\mathcal{E}_{k+1}\right)$ então, $\mathcal{E}_{k+1}<\|(T-\lambda I d)(y)\| \leq$ $\max _{i \in \mathbb{N}}\left|\alpha_{i}\right|=\mathcal{E}_{k}$, contradição. Logo $\mathcal{Y}_{\lambda}\left(\mathcal{E}_{k}\right) \subset \mathcal{Y}_{\lambda}\left(\mathcal{E}_{k+1}\right)$.

Agora provaremos que se $M$ é um subconjunto finito de $I(T)$, então para algum $\mathcal{E}>0$, os espaços $\left(\mathcal{Y}_{\lambda}(\mathcal{E})\right)_{\lambda \in M}$ formam uma soma direta, e então do fato de $X$ ser $H D_{n}$, segue claramente que $|I(T)| \leq n$.

Assumamos que a propriedade é falsa, isto é, para $M$ subconjunto finito de $I(T)$ de mínima cardinalidade entre aqueles contradizendo a propriedade, os espaços $\left(\mathcal{Y}_{\lambda}(\mathcal{E})\right)_{\lambda \in M}$ não formam soma direta, ou seja, $\forall \mathcal{E}>0, \mathcal{Y}_{\lambda_{1}}(\mathcal{E})+\cdots+\mathcal{Y}_{\lambda_{n}}(\mathcal{E})$ em $X$ não é uma soma direta. É claro que $|M| \geq 2$.

Seja agora a aplicação $T$ do subespaço $\mathcal{Y}_{\lambda_{1}}(\mathcal{E}) \oplus \cdots \oplus \mathcal{Y}_{\lambda_{n}}(\mathcal{E})$ para $\mathcal{Y}_{\lambda_{1}}(\mathcal{E})+\cdots+\mathcal{Y}_{\lambda_{n}}(\mathcal{E})$ definida por $T\left(y_{\lambda_{1}}, \cdots, y_{\lambda_{n}}\right)=y_{\lambda_{1}}+\cdots+y_{\lambda_{n}}$. Considere sobre o domínio a norma sup $\left\|y_{\lambda_{i}}\right\|$, $\forall\left(y_{\lambda_{1}}, \cdots, y_{\lambda_{n}}\right) \in D_{T}$. Então vejamos que :

- T é contínua pois :

$\left\|T\left(y_{\lambda_{1}}, \cdots, y_{\lambda_{n}}\right)\right\|=\left\|y_{\lambda_{1}}+\cdots+y_{\lambda_{n}}\right\| \leq\left\|y_{\lambda_{1}}\right\|+\cdots+\left\|y_{\lambda_{n}}\right\| \leq|M| \sup _{1 \leq i \leq n}\left\|y_{\lambda_{i}}\right\|, \forall y \in D_{T}$.

- $T$ é sobrejetora. De fato, seja $\alpha \in \mathcal{Y}_{\lambda_{1}}(\mathcal{E})+\cdots+\mathcal{Y}_{\lambda_{n}}(\mathcal{E})$. Daí temos $\alpha=y_{\alpha_{\lambda_{1}}}(\mathcal{E})+\cdots+y_{\alpha_{\lambda_{n}}}(\mathcal{E}) . \quad$ Assim considerando 0 elemento $\left(y_{\alpha_{\lambda_{1}}}, \cdots, y_{\alpha_{\lambda_{n}}}\right) \in \mathcal{Y}_{\lambda_{1}}(\mathcal{E}) \oplus \cdots \oplus \mathcal{Y}_{\lambda_{n}}(\mathcal{E})$, temos $T\left(y_{\alpha_{\lambda_{1}}}, \cdots, y_{\alpha_{\lambda_{n}}}\right)=\alpha$. 
- $T$ é injetora, pois dado $\left(y_{\lambda_{1}}, \cdots, y_{\lambda_{n}}\right) \in K \operatorname{KerT}$ temos que :

$T\left(y_{\lambda_{1}}, \cdots, y_{\lambda_{n}}\right)=y_{\lambda_{1}}+\cdots+y_{\lambda_{n}}=\sigma \Longrightarrow y_{\lambda_{1}}=-y_{\lambda_{2}}-\cdots-y_{\lambda_{n}}$. Mas por hipótese temos $Y_{\lambda_{1}}(\mathcal{E}) \cap\left(\mathcal{Y}_{\lambda_{2}}(\mathcal{E}) \oplus \cdots \oplus \mathcal{Y}_{\lambda_{n}}(\mathcal{E})\right)=\{\sigma\}$. Logo, $y_{\lambda_{1}}=\sigma=-y_{\lambda_{2}}-\cdots-y_{\lambda_{n}}$, e assim sucessivamente temos que $\operatorname{Ker}(T)=\{\sigma\}$, o que implica $T$ ser injetora.

Portanto, podemos afirmar que $T$ é contínua, injetora e sobrejetora.

Por outro lado, segue do resultado da Proposição 1.2 .25 que se $\left.\mathcal{Y}_{\lambda_{1}}(\mathcal{E})+\cdots+\mathcal{Y}_{\lambda_{n}} \mathcal{E}\right)$ não é direta, então $T: \mathcal{Y}_{\lambda_{1}}(\mathcal{E}) \oplus \cdots \oplus \mathcal{Y}_{\lambda_{n}}(\mathcal{E}) \longrightarrow \mathcal{Y}_{\lambda_{1}}(\mathcal{E})+\cdots+\mathcal{Y}_{\lambda_{n}}(\mathcal{E})$ não é um isomorfismo.

Uma vez que as afirmações acima são verdadeiras, segue que $T^{-1}:\left(\mathcal{Y}_{\lambda_{1}}(\mathcal{E})+\cdots+\right.$ $\left.\mathcal{Y}_{\lambda_{n}}(\mathcal{E})\right) \longrightarrow \mathcal{Y}_{\lambda_{1}}(\mathcal{E}) \oplus \cdots \oplus \mathcal{Y}_{\lambda_{n}}(\mathcal{E})$ definida por $T^{-1}\left(y_{\lambda_{1}}+\cdots+y_{\lambda_{n}}\right)=\left(y_{\lambda_{1}}, \cdots, y_{\lambda_{n}}\right)$ não é contínua. Logo não existe constante $K$ tal que $K\left\|\left(y_{\lambda_{1}}, \cdots, y_{\lambda_{n}}\right)\right\| \leq\left\|T\left(y_{\lambda_{1}}, \cdots, y_{\lambda_{n}}\right)\right\|$. Portanto, só podemos afirmar que $\forall \mathcal{E}>0,\left\|T\left(y_{\lambda_{1}}, \cdots, y_{\lambda_{n}}\right)\right\| \leq \mathcal{E}\left\|\left(y_{\lambda_{1}}, \cdots, y_{\lambda_{n}}\right)\right\|$, ou seja, $\frac{\left\|T_{y}\right\|}{\|y\|} \leq \mathcal{E}$.

Dessa maneira para os vetores $y_{\lambda} \in \mathcal{Y}_{\lambda}(\mathcal{E}), \underset{\lambda \in N}{\max }\left\|y_{\lambda}\right\|=1$ temos $\left\|\Sigma_{\lambda \in M} y_{\lambda}\right\| \leq \mathcal{E}$.

Aplicando $T$ na desigualdade abaixo, obtemos :

$\left\|\Sigma_{\lambda \in M} \lambda y_{\lambda}\right\|=\left\|\lambda_{1} y_{\lambda_{1}}+\cdots+\lambda_{n} y_{\lambda_{n}}\right\|=\| \lambda_{1} y_{\lambda_{1}}+\cdots+\lambda_{n} y_{\lambda_{n}}+T\left(y_{\lambda_{1}}\right)-T\left(y_{\lambda_{1}}\right)+\cdots+$ $T\left(y_{\lambda_{n}}\right)-T\left(y_{\lambda_{n}}\right)\|\leq\| T\left(y_{\lambda_{1}}\right)-\lambda_{1} y_{\lambda_{1}}\|+\cdots+\| T\left(y_{\lambda_{n}}\right)-\lambda_{n} y_{\lambda_{n}}\|+\| T\left(y_{\lambda_{1}}+\cdots+y_{\lambda_{n}}\right) \|=$ $|M| \mathcal{E}+\|T\| \cdot\left\|y_{\lambda_{1}}+\cdots+y_{\lambda_{n}}\right\| \stackrel{(1)}{\leq}|M| \mathcal{E}+\|T\| \mathcal{E}=(|M|+\|T\|) \cdot \mathcal{E}$.

Seja $c=\min _{\lambda \neq \lambda^{\prime} \in M}\left|\lambda-\lambda^{\prime}\right|$ e $C=\max _{\lambda \in M}|\lambda|$.

Seja $\lambda_{\mathcal{E}}$ em $M$ tal que $\max _{\lambda \neq \lambda_{\mathcal{E}}}\left\|y_{\lambda}\right\|=1$ (tal número existe porque $|M| \geq 2$ ). Então, temos:

$\left\|\Sigma_{\lambda \neq \lambda_{\mathcal{E}}}\left(\lambda-\lambda_{\mathcal{E}}\right) y_{\lambda}\right\|=\left\|\left(\lambda_{1}-\lambda_{\mathcal{E}}\right) y_{\lambda_{1}}+\cdots+\left(\lambda_{n}-\lambda_{\mathcal{E}}\right) y_{\lambda_{n}}\right\|=\| \lambda_{1} y_{1}-\lambda_{\mathcal{E}} y_{\lambda_{1}}+\cdots+\lambda_{n} y_{\lambda_{n}}-$ $\lambda_{\mathcal{E}} y_{\lambda_{n}}\|\leq\| \lambda_{1} y_{\lambda_{1}}+\cdots+\lambda_{n} y_{\lambda_{n}}\|+\|\left(-\lambda_{\mathcal{E}}\right)\left(y_{\lambda_{1}}+\cdots+y_{\lambda_{n}}\right) \| \stackrel{(1)}{\leq}(\|T\|+|M|) \cdot \mathcal{E}+\left|\lambda_{\mathcal{E}}\right| \mathcal{E}=$ $\left(\left|\lambda_{\mathcal{E}}\right|+\|T\|+|M|\right) \cdot \mathcal{E}$ enquanto $\max _{\lambda \neq \lambda_{\mathcal{E}}}\left\|\left(\lambda-\lambda_{\mathcal{E}}\right) y_{\lambda}\right\| \geq c=\min _{\lambda \neq \lambda^{\prime} \in M}\left|\lambda-\lambda^{\prime}\right|$.

Visto que $\mathcal{E} \longmapsto \mathcal{Y}_{\lambda}(\mathcal{E})$ é crescente para cada $\lambda$ fixado, e que $\lambda_{\mathcal{E}}$ torna $M \mathrm{com}$ um número finito de valores, podemos assumir que $\lambda_{\mathcal{E}}$ é constantemente igual para algum $\lambda_{0}$ tal 
que obtemos vetores $y_{\lambda}^{\prime}$ para $\lambda \neq \lambda_{0} \operatorname{com} y_{\lambda}^{\prime} \in \mathcal{Y}_{\lambda}(\mathcal{E}),\left\|\sum_{\lambda \neq \lambda_{0}} y_{\lambda}^{\prime}\right\| \leq(C+\|T\|+|M|) \cdot \mathcal{E}$, e $\max _{\lambda \neq \lambda_{0}}\left\|y_{\lambda}^{\prime}\right\| \geq c$.

Novamente, se $\mathcal{E} \longmapsto \mathcal{Y}_{\lambda}(\mathcal{E})$ é crescente, a soma de $\left(\mathcal{Y}_{\lambda}(\mathcal{E})\right)$ para $\lambda \neq \lambda_{0}$ não é direta, e isso mantém para algum $\mathcal{E}>0$ uma contradição com a minimalidade de $M$, pois ao diminuirmos os valores de $\mathcal{E}>0$ podemos obter um $M^{\prime}$ tal que $\left|M^{\prime}\right|<|M|$, onde $\left(\mathcal{Y}_{\lambda}\left(\mathcal{E}_{M^{\prime}}\right)\right)$ continua como uma soma não direta, o que contradiz com a suposição feita para $M$.

Finalmente estamos em condições de demonstrar o principal resultado deste capítulo, o Lema 6.3.1 que terá um papel fundamental na demonstração do Teorema Principal (Teorema 7.1.1).

\subsection{Operadores semi-Fredholm e Fredholm em espaços de Banach $H D_{n}$}

Lema 6.3.1 Seja X um espaço de Banach $H D_{n}$. Então todo operador semi-Fredholm sobre $X$ é Fredholm com índice zero.

Prova: Seja $T$ um operador semi-Fredholm sobre um espaço de Banach $X$. Consideremos primeiro o caso em que seja o espaço de Banach $X$ complexo $H D_{n}$. Então, da Proposição 6.2.4 $S(T)$ é quando muito um conjunto com $n$ pontos isolados.

Se $T$ é semi-Fredholm com $i(T)=\infty$, então pela continuidade do índice generalizado de Fredholm, para $\lambda \in S(T)$ temos que $(T-\lambda I d)$ é semi-Fredholm, o que contradiz o Lema 6.2.3 onde prova-se que $(T-\lambda I d)$ não é semi-Fredholm e nem Fredholm para $\lambda \in \delta S(T)=$ $S(T)$. Portanto $T$ é Fredholm.

Como $S(T)$ é finito, temos pela Definição 1.2.26 que o aberto $\mathbb{C} \backslash S(T)$ é conexo (por caminhos), e isso implica que $i(T-\lambda I d)$ é constantemente igual à $i(T)$ para $\lambda$ em $\mathbb{C} \backslash S(T)$ pela Proposição 6.2.1.

Além disso, quando $|\lambda| \longrightarrow \infty, T-\lambda I d=-\lambda(I d-T / \lambda)$ está próximo à $\lambda I d$, senão vejamos: 
Seja o operador $I d: X \longrightarrow X$, para o qual $i(I d)$ é definido, existe $\mathcal{E}>0$ tal que $\left(-\frac{T}{\lambda}\right): X \longrightarrow X$ satisfaz $\left\|-\frac{T}{\lambda}\right\|<\mathcal{E}$. Então, pela Proposição 1.5.3.10.(iii) temos que:

$$
i(T-\lambda I d)=i\left(I d-\frac{T}{\lambda}\right)=i(I d)=\alpha(I d)-\beta(I d)=0-0=0 .
$$

Portanto, $(T-\lambda I d)$ é Fredholm com índice zero, por consequência de continuidade $\operatorname{ind}(T)=0$.

Assumamos agora que $X$ é um espaço de Banach real $H D_{n}$ e considere sua complexificação $X_{\mathbb{C}}$.

Lembremos que $X_{\mathbb{C}}$, complexificação de $X$, é definida por $X_{\mathbb{C}}=X \oplus X$ com a lei $i(x, y)=(-y, x)$ (ver Definição 1.8.8).

Então pela Proposição 3.2.2 $X_{\mathbb{C}}$ é um espaço de Banach real $H D_{2 n}$ e como todo subespaço complexo de $X_{C}$ é também um subespaço real, temos que $X_{C}$ é um espaço de Banach complexo $H D_{m}$ para algum $m \leq 2 n$.

Seja $i_{T}$ o índice generalizado de Fredholm de $T$. A complexificação de $T$ sobre $X_{\mathbb{C}}$, $T \oplus T$ é também semi-Fredholm com índice $i_{T}$ (as dimensões na definição do índice de $T \oplus T$ são sobre $\mathbb{C})$.

Como $X_{\mathbb{C}}$ é hereditariamente finitamente decomponível e pela primeira parte dessa prova temos que $i_{T}$ é igual a zero.

Observação 6.3.2 Podemos obter por corolário, do Lema 6.3.1, que se X é um espaço de Banach hereditariamente finitamente decomponivel, entäo $X$ nào é isomorfo a nenhum subespaço próprio. 


\section{O TEOREMA PRINCIPAL}

Neste último capítulo faremos a demonstração do Teorema Principal (Teorema 7.1.1) de nossa dissertação. Sobre um espaço de Banach real hereditariamente indecomponível, o quociente do espaço de seus operadores pelo espaço de seus operadores estritamente singulares será caracterizado e sua dimensão limitada.

\subsection{Operadores em espaço de Banach real H.I.}

Na demonstração do Teorema Principal (Teorema 7.1.1) usaremos as Proposições 1.5.2.7, 1.8.15, 1.9.3.5 e os Lemas 2.4.1 e 6.3.1.

Teorema 7.1.1 Seja X um espaço de Banach real hereditariamente indecomponível. Então, para todo subespaço de dimensão infinita $Y$ de $X$, a dim $(L(Y, X) / S(Y, X)) \leq 4$. Além disso, $L(X) / S(X)$ é um anel de divisão isomorfo a $\mathbb{R}, \mathbb{C}$ ou $\mathbb{H}$.

Prova: Sejam $X$ um espaço de Banach real H.I., $Y$ um subespaço fechado de dimensão infinita de $X$, e $T \in L(Y, X)$. Denotamos $E_{Y}$ o espaço quociente de $L(Y, X)$ pelo Kernel da aplicação $\|\cdot\|_{Y}$ definida sobre $L(Y, X)$, isto é, por $\|T\|_{Y}=\underset{Z^{\prime} \subset Z}{\inf }\left\|T_{\left.\right|_{Z^{\prime}}}\right\|$ (veja a Observação 1.9.1.1.(ii)).

Segue da Observação 1.9.1.1 (i) que o kernel das aplicações de $L(Y, X)$ são operadores estritamente singulares. Dessa maneira $E_{Y}=L(Y, X) / S(Y, X)$, tal como na Definição 1.9.1.2.

Segue também que é possível construir a partir de $E_{Y}$ um espaço quociente $E$ com norma indicada na Definição 1.9.2.5 e completo (observação da Definição 1.9.3.3) tal que pela Observação 1.8.2.(iii), $E$ é uma Álgebra de Banach.

Por outro lado, com a multiplicação dada na Definição 1.9.3.1, E possui estrutura de álgebra normada com unidade (Proposição 1.9.3.2) e por todo elemento não nulo de $E$ ser invertível (observação da Definição 1.9.3.3) segue que $E$ é um anel de divisão. 
Uma vez que as afirmações acima são verdades, segue pelo Teorema 1.8.15, caso real do Teorema de Gelfand-Mazur, que $E$ é isomorfo ou a $\mathbb{R}$, ou a $\mathbb{C}$, ou a $\mathbb{H}$, e portanto que $\operatorname{dim} E \leq 4$.

Agora, como $E_{Y}$ é isomorfo a um subespaço de $E$ (veja Proposição 1.9.3.5), segue que a dimensão do espaço $E_{Y}$ é no máximo igual a 4 .

A afirmação relativa ao caso $Y=X$ pode ser provada diretamente como segue.

Seja $T$ um operador não estritamente singular sobre $X$. Então, pelo Lema 2.4.1, nenhuma restrição de $T$ para um subespaço de $X$ é estritamente singular.

Pela Proposição 1.5.2.7, a restrição de $T$ para algum subespaço de codimensão finita de $X$ é um isomorfismo na sua imagem, e portanto $T$ é semi-Fredholm.

Finalmente, pelo Lema 6.3.1, temos que $T$ é Fredholm.

Isso significa que todo operador sobre $X$ é ou estritamente singular ou Fredholm.

Como os operadores Fredholm são da classe dos operadores invertíveis do módulo dos operadores estritamente singulares, temos que isso é equivalente para dizer que $L(X) / S(X)$ é um anel de divisão, e portanto pelo Teorema de Gelfand-Mazur (Teorema 1.8.15) ele é isomorfo ou aos reais, ou aos complexos, ou ao anel dos quatérnios.

Finalmente observamos que o seguinte problema está em aberto :

Problema : Fixe $n \in \mathbb{N}^{*}$. Para todo $m \in \mathbb{N}^{*}, m \leq n^{2}$, existe $X_{m} H D_{n}$ tal que

$$
\operatorname{dim}\left(L\left(X_{m}\right) / S\left(X_{m}\right)\right)=m ?
$$




\section{REFERÊNCIAS BIBLIOGRÁFICAS}

[1] Bonsall F.F. e Ducan J., Complete Normed Algebras. Springer-Verlag, New York, 1973.

[2] Enflo, P., A counterexample to the approximation property in Banach spaces. Acta Math. 130 (1973), 309 - 317.

[3] Ferenczi, V., Operators on subspaces of hereditarily indecomposable Banach spaces. Bull. London Math. Soc. 29, (1996), 338 - 344.

[4] Ferenczi, V., Hereditarily finitely decomposable Banach spaces. Studia Mathematica 123 (2) (1997), 135 - 149.

[5] Ferenczi, V., Quotient hereditarily indecomposable Banach spaces. Canad. J. Math. 51 (3)(1999), 566-584.

[6] Galego, E.M., Alguns aspectos da teoria geométrica de espaços de Banach através de $c_{o}(\mathbb{N})$. 43‥ Seminário Brasileiro de Análise, IME-USP, 1996.

[7] Goldberg, S., Unbounded Linear Operators. Theory and Applications. McGraw-Hill, inc., 1966.

[8] Gowers, W.T., A new dichotomy for Banach spaces,

[9] Gowers, W.T. e Maurey, B., The inconditional basic sequence problem. J. Amer. Math. Soc. 6 (1993), 851 - 874. 
[10] Hönig, C.S., Análise Funcional e Aplicações. V.1, IME-USP, 1970.

[11] Hönig, C.S., Análise Funcional e Aplicações. V.2, IME-USP, 1970.

[12] Lindenstrauss, J e Tzafriri, L., Classical Banach Spaces, V.1, New York, Springer, 1977.

[13] Jesus, O.S., Subespaços Complementados na Soma de Espaços de Banach. IME-USP, 2002 (Dissertação de Mestrado).

[14] Semadeni, Z., Banach spaces of continous functions. PWN, Warszawa, 1971.

[15] Silva, R.R., Operadores em subespaços de espaços de Banach hereditariamente indecomponíveis. IME-USP, 2004 (Dissertação de Mestrado). 